Federal update contributes to the pediatric healthcare focus of this issue. It provides the rationale and recommendations on immunizations from Morbidity and Mortality Weekly Report, April 7, 1989, vol 38, No. 13. This update of a 1983 statement has many significant changes.

\title{
From the CDC
}

\section{Recommendations of the Immunization Practices Advisory Committee (ACIP)}

General recommendations on immunization

This revision of the "General recommendations on immunization" updates the 1983 statement. $^{1}$ Changes or new sections include: (1) listing of vaccines available in the United States by type and recommended routes; (2) updated schedules for immunizing infants and children; (3) clarification of the guidelines for spacing administration of immune globulin preparations and different vaccines; (4) an updated table of recommendations for routine immunization of children infected with human immunodeficiency virus; (5) listing of conditions that are often inappropriately considered contraindications to immunization; and (6) addition of information on the National Childhood Vaccine Injury Act of 1986 and the National Vaccine Injury Compensation Program. These recommendations are not comprehensive for each vaccine; Immunization Practices Advisory Committee (ACIP) recommendations on each vaccine should be consulted for more details.

\section{Introduction}

Recommendations for immunizing infants, children, and adults are based on characteristics of immunobiologics, scientific knowledge about the principles of active and passive immunization, and judgments by public health officials and specialists in clinical and preventive medicine. Benefits and risks are associated with the use of all immunobiologics: No vaccine is completely safe or completely effective. Benefits of immunization range from partial to complete protection against the consequences of disease (which range from mild or asymptomatic infection to severe consequences, such as paralysis or death); risks of immunization range from common, trivial, and inconvenient side effects to rare, severe, and life-threatening conditions. Thus, recommendations for immunization practices balance scientific evidence of benefits, costs, and risks to achieve optimal levels of protection against infectious diseases. These recommendations describe this balance and attempt to minimize the risks by providing specific advice regarding dose, route, and spacing of immunobiologics and delineating situations that warrant precautions or contraindicate their use. They are recommendations for use in the United States because epidemiologic circum- stances and vaccines often differ in other countries. Individual circumstances may warrant deviations from these recommendations. The relative balance of benefits and risks can change as diseases are controlled or eradicated. For example, because smallpox has been eradicated throughout the world, the risk of complications associated with smallpox vaccine now exceeds the risk of the disease; consequently, smallpox vaccination of civilians is now indicated only for laboratory workers directly involved with smallpox or closely related orthopox viruses (eg, monkeypox and vaccinia).

\section{Definitions}

\section{Immunobiologics}

Immunobiologics include both antigenic substances, such as vaccines and toxoids, and antibody-containing preparations, including globulins and antitoxins, from human or animal donors. These products are used for active or passive immunization or therapy. Examples include:

Vaccine: A suspension of live (usually attenuated) or inacti(continued on page 1196 


\section{SUPPLY $\uparrow$ DEMAND}

\section{ECG CHANGES}

\section{ANGINA}




\section{PROCARDIA (NIFEDIPINE) \\ PREVENTS THE PAIN... \\ Protects THE Angina Patient AT EVERYSTAGE}

SUPPLYIDEMAND - PROCARDIA increases myocardial oxygen supply and reduces demand

LV Function O PROCARDIA helps to maintain or enhance parameters of left ventricular function'

ecG Changes

- PROCARDIA decreases the incidence of ischemic ECG changes ${ }^{2}$

ANGINA

- PROCARDIA reduces episodes of both painful and painless ischemia ${ }^{3.4}$

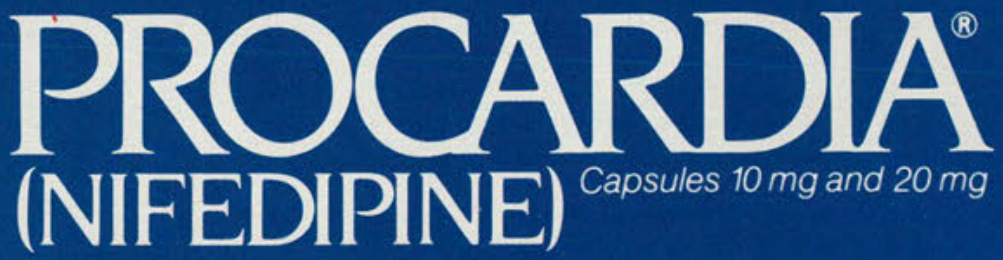

Prevents The Pain...

Protects the Angina Patient

Please see PROCARDIA ${ }^{\circledast}$ (nifedipine) brief summary on following page. 


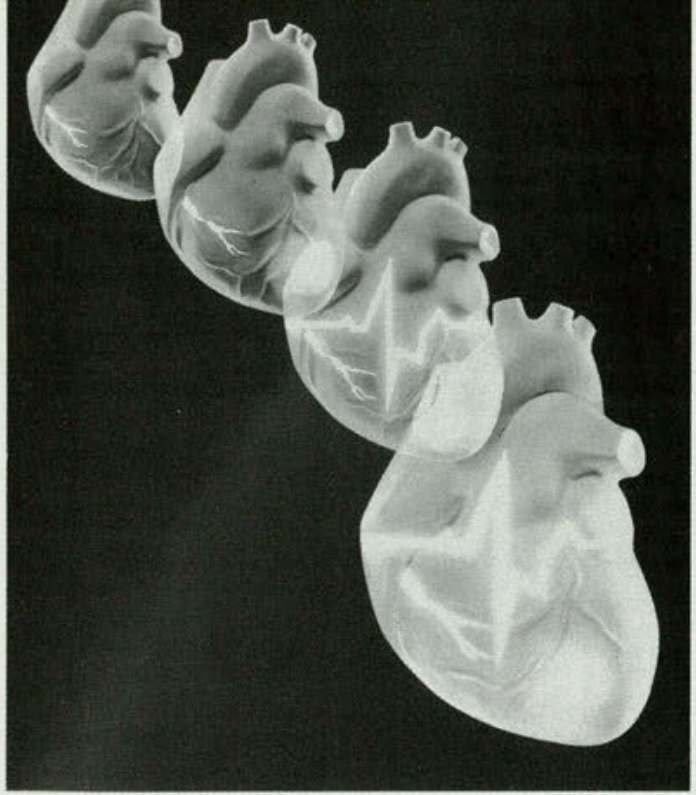

In the evolution of an angina attack, PROCARDIA PreventS THE PAin... Protects the Angina PAtient

\section{References:}

1. Vetrovec GW, Parker VE: Acute electrophysiologic, hemodynamic and left ventricular effects of nifedipine and beta-blocker interactions Maintenance of global and

2. Oakley GDG. Fox KM. Dargie HJ, et al: Objective assessment of treatment in severe angina. Br Med J 1979:2:1540.

3. Stone PH. Muller JE. Turi ZG. et al: Efficacy of nifedipine therapy in patients with refractory angina pectoris: Significance of the presence of coronary vasospasm. Am Heart J 1983: 106-644-652

4. Data on file. Medical Department, Pfizer Laboratories, Pfizer Inc. New York
...With an Excellent Side-Effects Profile

PROCARDIA side effects are usually mild and include dizziness, peripheral edema, nausea, weakness, headache and flushing (each in about $10 \%$ of patients); transient hypotension (about $5 \%$ ); exacerbation of angina occurs rarely.

\section{Brief Summary}

INDICATIONS (nifedipine) Capsules sospastic angina contirmed by any of the following criteria: 1) classical pattern of angina at rest accompanied by ST segment elevation, 2) angina or coronary artery spasm provoked by ergonovine, or 3) angiographically demonstrated
coronary artery spasm. In those patients who have had angiography, the presence of significant fixed obstructive disease is not incompatible with the diagnosis of vasospastic angina, provided that the above criteria are satisfied. PROCARDIA may also be used where the clinical presentation suggests a possible vasospastic component but where vasospasm has not been confirmed, e.g. where pain has a variable threshold on exertion or in unstable angina where electrocardiographic findings are compatible with intermittent vasospasm, or when angina is refractory to nitrates and/or adequate doses of beta blockers

II. Chronic Stable Angina (Classical Effort-Associated Angina): PROCARDIA is indicated for the management of chronic stable angina (efflort-associated angina) without evidence of vasospasm in patients who re
spite adequate doses of beta blockers and/or organic nitrates or who cannot tolerate those agents.

spite adequate doses of beta blockers and/or or ganic nitrates or who cannot tolerate those agents. weeks duration in reducing angina trequency and increasing exercise tolerance, but confirmation of sustained effective. ness and evaluation of long-term safety in these patients are incomplete.

Controlled studies in small numbers of patients suggest concomitant use of PROCARDIA and beta-blocking agents may be beneficial in patients with chronic stable angina, but available information is not sufficient to predict with confidence the effects of concurrent treatment, especially in patients with compromised left ventricular function or cardiac conduction abnormalities. When introducing such concomitant therapy, care must be taken to monitor blood pressure closely since severe hypotension can occur from the combined effects of the drugs. (See WARNINGS.)

CONTRAINDICATIONS: Known hypersensitivity reaction to PROCARDIA.

WARNINGS: Excessive Hypotension: Although in most patients, the hypotensive effect of PROCARDIA is modest and weil tolerated, occasional pattenis have had excessive and pooriy tolerated hypolension. These responses have usually Occuremis

Severe hypotension and/or increased fluid volume requirements have been reported in patients receiving PROCARDIA The interaction with high dose fentanyl appears to be due to the combination of PROCARDIA and a beta blockes, but the possibility that it may occur with PROCARDIA alone, with low doses of fentanyl, in other surgical procedures. or with other narcotic analgesics cannot be ruled out. In PROCARDIA treated patients where surgery using high dose fentanyl anesthesia is contemplated, the physician should be aware ort these potential problems and, if the patient's condition permits, sulficient time (at least 36 hours) should be allowed for PROCARDIA to be washed out of the body prior to surgery.

Increased Angina and/or Myocardial Infaretion: Rarely, patients, particularly those who have severe obstructive co onary artery disease. have developed well documented increased frequency. duration and/or severity of angina or acute myocardial intarction on starting PROCARDIA or at the time of dosage increase. The mechanism of this effect is not Beta Blocker Withdrawal: Patients recently withdrawn from beta blockers may develop a withdrawal syndrome with increased angina, probably related to increased sensitivity to catecholamines. Initiation of PROCARDIA treatment will not occasional reports of increased angina in a setting of beta blocker withdrawal and PROCARDIA initiation. It is important to taper beta blockers if possible, rather than stopping them abruptly before beginning PROCARDIA

Congestive Heart Failure: Rarely. patients, usually receiving a beta blocker, have developed heart failure after beginning PROCARDIA Patients with tight aortic stenosis may be at greater risk lor such an event

PRECAUTIONS: General: Hypotension: Because PROCARDIA decreases peripheral vascular resistance, careful moniloring of blood pressure during the initial administration and titration of PROCARDIA is suggested. Close observation is especially recommended tor patients already taking medications that are known to lower blood pressure. (See.

Peripheral edema: Mild to moderate peripheral edema, typically associated with arterial vasodilation and not due to left ventricular dystunction, Occurs in about one in ten patients treated with PROCARDIA. This edema occurs primarily in heart tailure, care should be taken to differentiate this peripheral edema trom the ettects of increasing oy congestive

dystunction. Laboratory tests: Rare, usUally transient, but occasionally significant elevations of enzymes such as alkaline phos -
phatase. CPK. LDH. SGOT and SGPT have been noted. The relationship to PROCARDIA therapy is uncertain in most cases, but probable in some. These laboratory abnormalities have rarely been associated with clinical symptoms, however, cho-

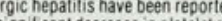

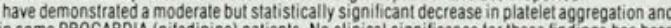
increase in bleeding time in some PROCARDIA (nitedipine) patients. No clinical significance for these findings has been demonstrated. Positive direct Coombs test with/without hemolytic anemia has been reported.

Although PROCARDIA has been used sately in patients with renal dystunction and has been reported to exert a beneficial effect incertain cases, rare, reversible elevations in BUN and serum creatinine have been reported in patients with preexisting chronic renal insufficiency. The relationship to PROCARDIA therapy is uncertain in most cases but probable in

Drug interactions: Beta-adrenergic blocking agents: (See INDICATIONS and WARNINGS.) Experience in over $1400 \mathrm{pa}$ lients in a non-comparative clinical trial has shown that concomitant administration of PROCARDIA and beta-blocking agents is usually well tolerated, but there have been occasional literature reports suggesting that the combination may in Leting

等 Devalis administratinal ellectiveness or this combination.

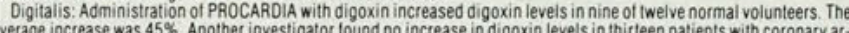
ery disease. In an uncontrolled study of over two hundred patients with congestive heart failure during which digoxin blood levels were not measured, digitalis toxicity was not observed. Since there have been isolated reports of patients with elevated digoxin levels, it is recommended that digoxin levels be monitored when initiating. adjusting, and discontinuing PROCARDIA to avoid possibie over- or under-digitalization.

Coumarin anticoagulants. There have been rare reports of increased prothrombin time in patients taking coumarin anicoagulants to whom PROCARDIA was administered.

Cimetidine: A study in six healthy volunteers has shown a significant increase in peak nifedipine plasma levels $(80 \%)$ and area-under-the-curve $(74 \%$ ) atter a one week course of cimetidine at $1000 \mathrm{mg}$ per day and nifedipine at $40 \mathrm{mg}$ per day cimetidine cautious titration is adviged.

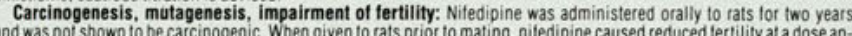
proximately 30 times the maximum recommended human dose. In vivo mutagenicity studies were negative

Pregnancy: Pregnancy Category C. Nifedipine has been shown to be teratogenic in rats and embryotoxic in rats mice and rabbits. There are no adequate and well controlled studies in pregnant women. PROCARDIA should be used during pregnancy only if the potential benefit justifies the potential risk to the fetus.

作 sea. weakness, headache and tlushing, each occurring in about $10 \%$ of patients, Iransient hypotension in about $5 \%$. paiitation in about $2 \%$ and syncope in about $0.5 \%$. Syncopal episoces did not recur with reduction in the dose of ROCARDIA or concomitant antianginal medication. Additionally, the lollowing have been reported: muscle cramps, nervousness, dyspnea, nasal and chest congestion, shortness of breath, diarthea. constipation, gasitrointestinal cramps, flat-

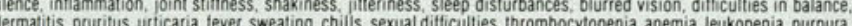
allergic hepatitis, erythromelalgia, and arthritis with ANA $(+)$ Very rarely introduction of PROCAROIA therapy was associated with an in crease in anginal pain, possibly due to associated hypotension.

In addition, more serious adverse events were observed, not readily distinguishable from the natural history of the dis. ease in these patients. It remains possible, however, that some or many of these events were drug related Myocardial in. arction occurred in about $4 \%$ of patients and congestive heart failure or pulmonary edema in about $2 \%$. Ventricular arrthythmias or conduction disturbances each occurred in fewer than $0.5 \%$ of patients

HOW SUPPLIED: PROCARDIA soft gelatin capsules are supplied in:

Bottles of $100: 10 \mathrm{mg}$ (NDC $0069-2600-66$ ) orange \#260;20 mg (NDC 0069-2610-66) orange and light brown \#261. Bottles of $300: 10 \mathrm{mg}$ ( (NDC 0069-2600-72) orange \#260:20 mg (NDC 0069-2610-72) orange and light brown \#261. Unit dose packages of 100: $10 \mathrm{mg}$ (NDC 0069-2600-41) orange \#260;20 mg (NDC 0069-2610-41) orange and light brown
\#261. The capsules should be protected from light
$25^{\circ} \mathrm{C}$ ) in the manulacturer's original container.

More detailed professional information available on request 


\section{The National Osteopathic Foundation Announces:}

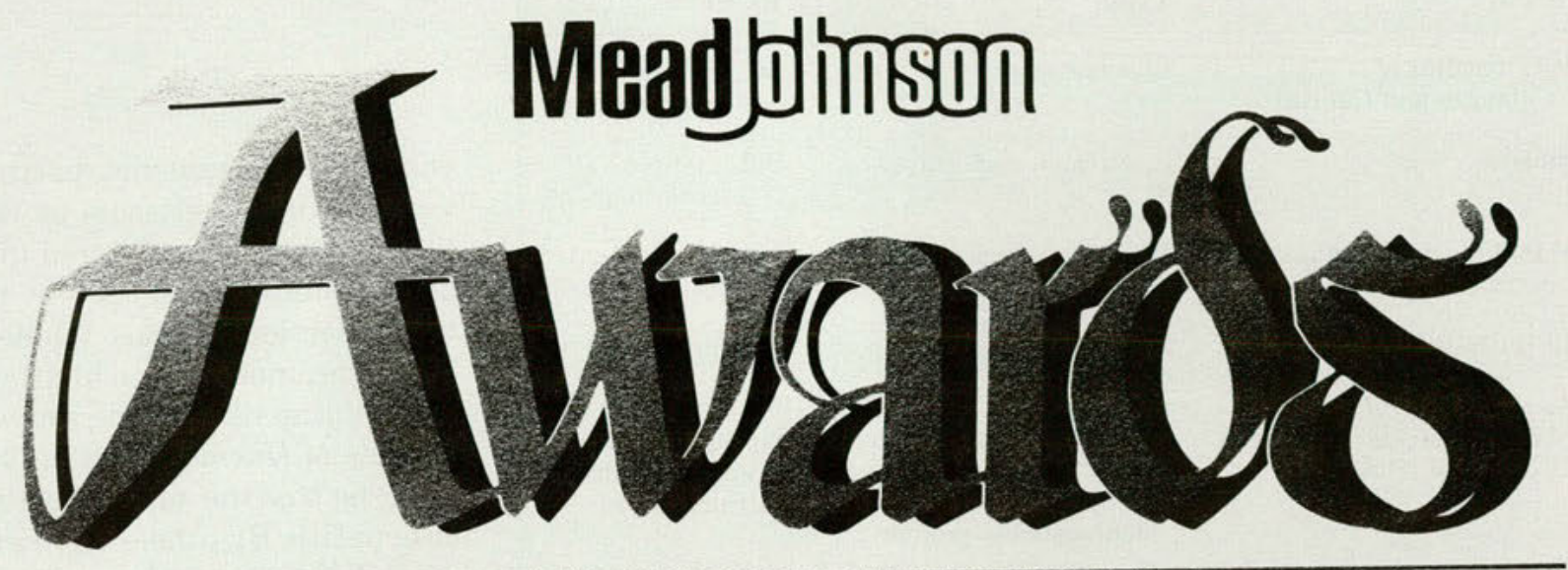

\section{FOR GRADUATE TRAINING}

Six $\$ 5,000$ awards in 1990 plus round trip transportation to AOA annual meeting.

Award recipients will be the guests of honor at an awards

breakfast to be hosted by Mead Johnson at the AOA Annual Meeting

For residency training in $\mathrm{AOA}$ approved specialties

The National Osteopathic Foundation administers all funds and selection of recipients

The Mead Johnson Awards for Graduate Training in osteopathic medicine provide financial assistance to selected graduates toward completion of a year of residency training. Mead Johnson has participated by providing funds for this joint endeavor since 1956.

Grants are available to any osteopathic physician within four years of date of graduation from an osteopathic college. The recipient may choose any hospital approved for residency training by the American Osteopathic Association.

Application forms may be obtained by writing to:

Committee on Educational Grants

The National Osteopathic Foundation

142 E. Ontario, 2nd Floor

Chicago, Illinois 60611

(800) 621-1773 (Ext. 5850)

\section{FORMS MUST BE RETURNED BY APRIL 15, 1990}


Table 1

Vaccines Available in the United States, by Type and Recommended Routes of Administration

\begin{tabular}{|c|c|c|}
\hline Vaccine & Type & Route \\
\hline $\begin{array}{l}\text { BCG (bacillus of } \\
\text { Calmette and Guérin) }\end{array}$ & Live bacteria & $\begin{array}{l}\text { Intradermal or } \\
\text { subcutaneous }\end{array}$ \\
\hline Cholera & Inactivated bacteria & $\begin{array}{l}\text { Subcutaneous or } \\
\text { intradermal* }\end{array}$ \\
\hline $\begin{array}{l}\text { DTP (diphtheria, tetanus, } \\
\text { pertussis) }\end{array}$ & $\begin{array}{l}\text { Toxoids and inactivated } \\
\text { bacteria }\end{array}$ & Intramuscular \\
\hline HB (hepatitis B) & Inactive viral antigen & Intramuscular \\
\hline $\begin{array}{l}\text { Haemophilus influenzae b } \\
\text { Polysaccharide }(\mathrm{HbPV}) \text { or } \\
\text { Conjugate (HbCV) }\end{array}$ & $\begin{array}{l}\text { Bacterial polysaccharide } \\
\text { or polysaccharide } \\
\text { conjugated to protein }\end{array}$ & $\begin{array}{l}\text { Subcutaneous or } \\
\text { intramuscular }{ }^{\dagger} \\
\text { Intramuscular }\end{array}$ \\
\hline Influenza & $\begin{array}{l}\text { Inactivated virus or } \\
\text { viral components }\end{array}$ & Intramuscular \\
\hline $\begin{array}{l}\text { IPV (inactivated } \\
\text { poliovirus vaccine) }\end{array}$ & $\begin{array}{c}\text { Inactivated viruses } \\
\text { of all } 3 \text { serotypes }\end{array}$ & Subcutaneous \\
\hline Measles & Live virus & Subcutaneous \\
\hline Meningococcal & $\begin{array}{l}\text { Bacterial polysaccharides } \\
\text { of serotypes } \\
\text { A/C/Y/W-135 }\end{array}$ & Subcutaneous \\
\hline $\begin{array}{l}\text { MMR (measles, mumps, } \\
\text { rubella) }\end{array}$ & Live viruses & Subcutaneous \\
\hline Mumps & Live virus & Subcutaneous \\
\hline $\begin{array}{l}\text { OPV (oral poliovirus } \\
\text { vaccine) }\end{array}$ & $\begin{array}{l}\text { Live viruses of all } 3 \\
\text { serotypes }\end{array}$ & Oral \\
\hline Plague & Inactivated bacteria & Intramuscular \\
\hline Pneumococcal & $\begin{array}{l}\text { Bacterial polysaccharides } \\
\text { of } 23 \text { pneumococcal } \\
\text { types }\end{array}$ & $\begin{array}{l}\text { Intramuscular or } \\
\text { subcutaneous }\end{array}$ \\
\hline Rabies & Inactivated virus & $\begin{array}{l}\text { Subcutaneous or } \\
\text { intradermal } \$\end{array}$ \\
\hline Rubella & Live virus & Subcutaneous \\
\hline Tetanus & Inactivated toxin (toxoid) & Intramuscular§ \\
\hline $\begin{array}{l}\text { Td or DT } \| \\
(\mathrm{T}=\text { tetanus; } \mathrm{D} \text { or } \\
\mathrm{d}=\text { diphtheria })\end{array}$ & $\begin{array}{l}\text { Inactivated toxins } \\
\text { (toxoids) }\end{array}$ & Intramuscular§ \\
\hline Typhoid & Inactivated bacteria & Subcutaneous \\
\hline Yellow fever & Live virus & Subcutaneous \\
\hline \multicolumn{3}{|c|}{$\begin{array}{l}\text { * The intradermal dose is lower. } \\
\text { Toute depends on the manufacturer; consult package insert for recommendation for specific } \\
\text { product used. } \\
\text { Intradermal dose is lower and used only for preexposure vaccination. } \\
\$ \text { Preparations with adjuvants should be given intramuscularly } \\
\text { ॥ DT = tetanus and diphtheria toxoids for use in children aged }<7 \text { years. Td }=\text { tetanus and } \\
\text { diphtheria toxoids for use in persons age } \geqslant 7 \text { years. Td contains the same amount of tetanus toxoid } \\
\text { as DTP or DT but a reduced dose of diphtheria toxoid. } \\
\text { I Boosters may be given intradermally unless acetone-killed and dried vaccine is used. }\end{array}$} \\
\hline
\end{tabular}

vated microorganisms (bacteria, viruses, or rickettsiae) or fractions thereof administered to induce immunity and thereby prevent infectious disease (Table 1). Some vaccines contain highly defined antigens (eg, the polysaccharide of Haemophilus influen$z a e$ type $\mathrm{b}$ or the surface antigen of hepatitis B); others have antigens that are complex or incompletely defined (eg, killed Bordetella pertussis or live attenuated viruses).

Toxoid: A modified bacterial toxin that has been rendered nontoxic but retains the ability to stimulate the formation of antitoxin.

Immune globulin (IG): A sterile solution containing antibodies from human blood. It is obtained by cold ethanol fractionation of large pools of blood plasma and contains $15 \%$ to $18 \%$ protein. Intended for intramuscular administration, it is primarily indicated for routine maintenance of immunity of certain immunodeficient persons and for passive immunization against measles and hepatitis A. IG does not transmit hepatitis B virus, human immunodeficiency virus (HIV), or other infectious diseases.

Intravenous immune globulin (IGIV): A product derived from blood plasma from a donor pool similar to the IG pool but prepared so it will be suitable for intravenous use. IGIV does not transmit infectious diseases. It is primarily indicated for replacement therapy in antibody-deficiency disorders.

Specific IG: Special preparations obtained from blood plasma (continued on page 1199) 


\section{Announcing a new, proven way to reduce the risk of coronary heart disease}

LOPID is now indicated for reducing the risk of coronary heart disease (CHD) in Type Ilb patients with low HDL*-cholesterol, elevated $L D L^{*}$-cholesterol and triglycerides, and who have had an inadequate response to weight loss, diet, exercise, and other pharmacologic agents such as bile acid sequestrants and nicotinic acid. The potential benefit of gemfibrozil in treating Type Ila patients who have elevations of LDL-cholesterol as their only lipid abnormality is not likely to outweigh the risks.

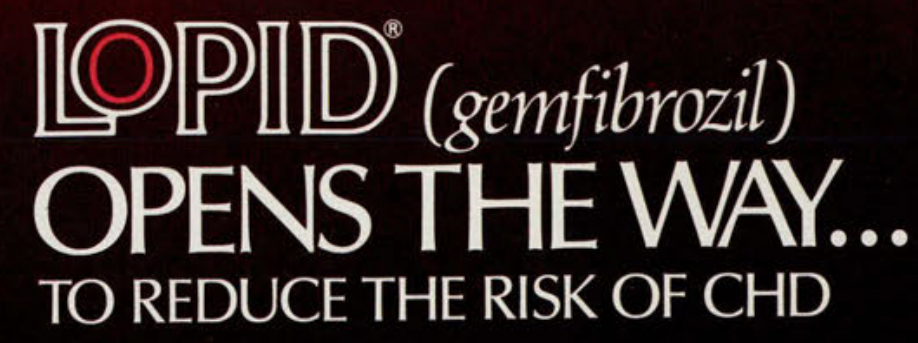


Lopid" (Gemfibrozil Capsules and Tablets)

\section{Before prescribing, please see full prescribing information.}

\section{A Brief Summary follows.}

CLINICAL PHARMACOLOGY. Lopid (gemfibrozil capsules and tablets) is a lipid regulating terol, and increases high density liperides and very low density ipoprotein (VLDL) choles otal and low density lipoprotein (LDL) cholesterol may be observed with Lopid therap treatment ol patients with elevated triglycerides due to Type IV hyperlipoproteinemia often results in a rise in LDL.cholesterol. LDL-cholesterol levels in Type llb patients with elevations of both serum LDL-cholesterol and triglycerides are, in general, minimally affected by Lopid treatment: however, Lopid usually raises HDL-cholesterol significantly in this group. Lopid increases levels of high density lipoprotein (HDL) subfractions $\mathrm{HDL}_{2}$ and $\mathrm{HDL}_{3}$, as well as apolipoproteins Al and All. Epidemiological studies have shown that both low HDL.cholesIn the high LDL-cholesterol are independent risk factors for coronary heart disease. primary prevention trial in 4081 male patients between the ages of 40 and 55 , Lopid therapy was associated with significant reductions in total plasma triglycerides and a significan increase in high density lipoprotein cholesterol. Moderate reductions in total plasma cholesterol and low density lipoprotein cholesterol were observed for the Lopid treatment group as a whole, but the lipid response was heterogeneous, especially among different Fredrickson Types. The study involved subjects with serum non-HDL-cholesterol of over $200 \mathrm{mg} / \mathrm{dL}$ an group experienced a $34 \%$ reduction in serious coronary events (sudden cardiac death plus fatal and nonfatal myocardial infarctions) compared to placebo. There was a 370 eduction in nonfatal myocardial infarction. The greatest reduction in the incidence of serio coronary events occurred in Type llb patients who had elevations of both LDL-cholesterol

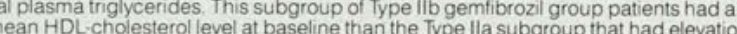
of $\mathrm{LDL}$-cholesterol and normal plasma triglycerides. The mean increase in $\mathrm{HDL}$-cholesterol among the Type lib patients in this study was $12.6 \%$ compared to placebo. It is not clear to what extent the findings of the Helsinki Heart Study can be extrapolated to other segments of the dyslipidemic population not studied or to other lipid-altering drugs

The mechanism of action has not been definitively established. In man. Lopid has been shown to inhibit peripheral lipolysis and to decrease the hepatic extraction of free fatty acids, thus reducing hepatic triglyceride production. Lopid inhibits synthesis and increases clearance of VLDL carrier apolipoprotein B, leading to a decrease in VLDL production. Animal studies suggest that Lopid may, in addition to elevating $\mathrm{HDL}$-cholesterol, reduce incorporation of long-chain fatty acids into newly formed triglycerides, accelerate turnover Lopid is well absorbed from the gastrointestinal tract after oral administration. Peak plasma levels occur in one to two hours with a plasma half-life of 1.5 hours following multiple doses. Plasma levels appear proportional to dose and do not demonstrate accumulation across time following multiple doses

methyl and a carboxyl metabolite. Approximately seventy percent of the administered human dose is excreted in the urine mostly as the excreted as unchanged gemfibrozil. Six percent of the dose is accounted for in the feces. CATIONS. 1. Hepatic or severe renal dysfunction, including primary biliary

\section{Preexisting gallbladder disease (See WARNINGS)}

WARNINGS, 1 Because of chem

Because or chemical, pharmacological, and clinical similarities between gemfibrozil and clofibrate, the adverse findings with clofibrate in two large clinical studies may also apply to gemtibrozil. In the first of those studies, the Coronary Drug Project, 1000 subjects with previous myocardial infarction were treated for five years with clofibrate. There treated subjects, but twice as many ciofibrate-treated subjects devels and 3000 placebocholecystitis requiring surgery. In the other study, conducted by the World Health Organi. zation (WHO), 5000 subjects without known coronary heart disease were treated with clofibrate for five years and followed one year beyond. There was a statistically significant. $29 \%$, higher total mortality in the clofibrate-treated than in a comparable placebo-treated control group. The excess mortality was due to a $33 \%$ increase in noncardiovascular causes, of clofibrate-treated subiects for gallbladder disease was confirmed

During the Helsinki Heart Study and in the $11 / 2$ year follow-up period since the trial was placebo group. Mortality from any cause during the double-blind portion of the study was 44 deaths in the Lopid group and 43 in the placebo group. Because of the more limited size of deaths in the Lopid group and 43 in the placebo group. Because of the more limited size
the Helsinki Heart Study. this result is not statistically-significantly different from the $29 \%$ the Helsinki Heart Study, this result is not statistically-significantly different from the $29 \%$
excess mortality seen in the clofibrate group in the separate WHO study. Noncoronary hear disease related mortality showed a $58 \%$ greater trend in the Lopid group ( 43 vs 27 patients the placebo group, $p=0.056$ )

In the Helsinki Heart Study, the incidence of total malignancies discovered during the trial and in the $11 / 2$ years since the trial was completed was 39 in the Lopid group and 29 in the placebo group (difference not statistically significant). This includes 5 basal cell carcinomas in the Lopid group and none in the placebo group $(\mathrm{p}=0.06$; historical data predicted an were not statistically different between Lopid and placebo subgroups. Follow-up of the Helsinki Heart Study participants will provide further information on cause-specific mortality cancer morbidity.

2. A gallstone prevalence substudy of 450 Helsinki Heart Study participants showed a group ( $7.5 \%$ vs $4.9 \%$ for the placebo group, a $55 \%$ excess for the gemfibrozil group). A trend
grend toward a greater incidence of gallbladder surgery was observed for the Lopid group (17 vs dence of cholecystectomy observed in the WHO study in the group treated with clofibrate. Both clofibrate and gemfibrozil may increase cholesterol excretion into the bile leading to cholelithiasis. If cholelithiasis is suspected, gallbladder studies are indicated. Lopid therapy

3. Since a reduction of mortality from coronary artery disease has not been demonstrated be administered only to those patients described in the INDICATIONS AND USAGE section. If a significant serum lipid response is not obtained, Lopid should be discontinued. 4. Concomitant Anticoagulants - Caution should be exercised when anticoagulants are given in conjunction with Lopid. The dosage of the anticoagulant should be reduced to

Frequent prothrombin determinations are advisable until it has been definitely determined that the prothrombin level has stabilized

5. Concomitant therapy with Lopid and Mevacor" (Iovastatin) has been associated with
habdomyolysis, markedly elevated creatine kinase (CK) levels and myoglobinuria. leading h a high proportion of cases to acute renal failure. In most subjects who have had an with lovastatin and remfonse to either drug alone, the possible benefit of combined therapy with lovastatin and gemfibrozil does not outweigh the risks of severe myopathy, rhabdom
lysis, and acute renal failure (See Drug Interactions). The use of fibrates alone, including Lopid, may occasionally be associated with myositis. Patients receiving Lopid and complain ing of muscle pain, tenderness, or weakness should have prompt medical evaluation for myositis, including serum creatine kinase level
diagnosed. Lopid therapy should be withdrawn.

6. Cataracts - Subcapsular bilateral cataracts occurred in $10 \%$, and unilateral in $6.3 \%$ of male rats treated with gemfibrozil at 10 times the human dose
PRECAUTIONS. 1. Initial Therapy - Laboratory stues sho the lipid levels are consistently abnormal. Before instituting Lould be done to ascertain that should be made to control serum lipids with appropriate diet, exercise. weight loss in obese patients, and control of any medical problems such as diabetes mellitus and hypothyroidism 2 Continued Therapy-Periodic determin
Lopid" (Gemfibrozil Capsules and Tablets)

drug withdrawn if lipid response is inadequate after 3 months of therapy

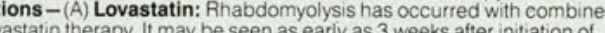
gemfibrozil and lovastatin therapy, It may be seen as early as 3 weeks after initiation of lipid response to either drug alone, the possible benefit of combined therapy with lovastatin and gemfibrozil does not outweigh the risks of severe myopathy, rhabdomyolysis, and acute renal falure. There is no assurance that periodic monitoring of creatine kinase will prevent (B) Anticoagulants: CAUTION SHOULD BE EXERC SHOULD BE REDUCED TO MAINTAIN THE PROTHROMBIN TIME AT THE DESIRED LEVEL TO PREVENT BLEEDING COMPLICATIONS. FREQUENT PROTHROMBIN DETER

PROTHROMBIN

4. Carcinogenesis, Mutagenesis, Impairment of Fertility-Long-term studies h. ve been conducted in rats and mice at one and ten times the human dose. The incidence of
benign liver nodules and liver carcinomas was significantly increased in high dose male rats. The incidence of liver carcinomas increased also in low dose males, but this increase was no statistically significant $(p=0.1)$. In high dose female rats, there was a significant increase in the combined incidence of benign, and malignant liver neoplasms. In male and female mice, but the doses tested were lower than those shown to be carcinogenic with other fibrates Male rats had a dose-related and statistically significant increase of benign Leydig ce umors at 1 and 10 times the human dose.

Electron microscopy studies have demonstrated a florid hepatic peroxisome proliferation following Lopid administration to the male rat. An adequate study to test for peroxisome proliferation has not been done in humans but changes in peroxisome morphology have two other drugs of the fibrate class when liver biopsies were compared before and after eatment in the same individual.

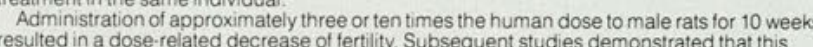
resulted in a dose-related decrease of fertility. Subsequent studies demonstrated that this effect was reversed after a drug-free period of about eight weeks, and it was not transmitted

Pregnancy Category B - Reproduction studies have been performed in the rat at doses 3 and 9 times the human dose, and in the rabbit at 2 and 6.7 times the human dose. These studies have revealed no evidence of impaired fertility in females or harm to the fetus due to Lopid. Minor fetotoxicity was manifested by reduced birth rates observed at the high
dose levels. No significant malformations were found among almost 400 offspring from 36 litters of rats and 100 fetuses from 22 litters of rabbits.

There are no studies in pregnant women. In view of the fact that Lopid is tumorigenic in
male and female rats, the use of Lopid in pregnancy should be reserved for those patients male and female rats, the use of Lopid in pregnancy should be reserved for

where the benefit clearly outweighs the possible risk to the patient or fetus.
. Nursing Mothers-Because of the potential for tumorigenicity shown for gemfibrozi in

rats, a decision should be made whether to discontinue nursing or discontinue the drug.

aking into account the importance of the drug to the mother

Hematologic Changes - Mild hemoglobin, hematocrit and white blood cell decreases have been observed in occasional patients following initiation of Lopid therapy. Howeve these levels stabilize during long-term administration. Rarely, severe anemia, leukopenia, thrombocytopenia, and bone marrow hypoplasia have been reported. Therefore, per
blood counts are recommended during the first 12 months of Lopid administration.

blood counts are recommended during the first 12 months of Lopid administration.
. Function - Abnormal liver function tests have been observed occasionally durin Lopid administration, including elevations of AST (SGOT). ALT (SGPT). LDH, bilirubin, and alkaline phosphatase. These are usually reversible when Lopid is discontinued. Therefore periodic liver function studies are recommended and Lopid therapy should be terminated if

\section{Use in Childre}

( 046 patients r were statistically more Lropid tor up to 5 years. In that study, the following adverse reaction theses): gastrointestinal reactions. $34.2 \%(23.8 \%)$; dyspepsia. $196 \%(119 \%)$; abdominal pain, $9.8 \%(5.6 \%)$; acute appendicitis, $1.2 \%(0.6 \%)$ : atrial fibrillation, $0.7 \%(0.1 \%)$.

Adverse events reported by more than $1 \%$ of subjects, but without a significant difference between groups (placebo incidence in parentheses) were: diarrihea, $7.2 \%(65 \%)$; fatigue. vertigo, $1.5 \%$ (1.3\%); constipation, $1.4 \%(1.3 \%)$; headache, $1.2 \%(1.1 \%)$

Gallbladder surgery was performed in $0.9 \%$ of Lopid and $0.5 \%$ of placebo subjects, a $64 \%$ excess, which is not statistically different from the excess of gallbladder surgery observed the clofibrate compared to the placebo group of the WHO study.

Nervous system and special senses adverse reactions were more common in the Lopid group. These included hypesthesia, paresthesias, and taste perversion. Other adverse reactions that were more common among Lopid treatment group subjects but where a causal relationship was not established include cataracts, peripheral vascular disease, and intracerebral hemorrhage.

musculoskeletal symptoms (See WARNINGS), and to abnormal liver function tests an hematologic changes (See PRECAUTIONS) Reports of viral and bacterial infections (common cold, cough, urinary tract infections) were Additional adverse reactions that have been reported for gemfibrozil are listed below by system. These are categorized according to whether a causal relationship to treatment with Lopid is probable or not established:

CAUSAL RELATIONSHIP PROBABLE: Gastrointestinal: cholestatic jaundice; Central Nervous System: dizziness, somnolence, paresthesia, peripheral neuritis, decreased libido. myopathy, myasthenia, myalgia, painful extremities, arthral imia, synovitis, rhabdomyolysis (see WARNINGS and Drug Interactions under PRECAUTIONS); Clinical Laboratory: increased creatine phosphokinase, increased bilirubin, increased liver transaminases (AST bone marrow hypoplasia, eosinophilia; /mmunologic: angioedema, laryngeal edema. Urticaria; Integumentary: exfoliative dermatitis, rash, dermatitis, pruritus. sions, syncope: Eye: retinal edema; Genitourinary: decreased male fertility: Clinical Labora ory. positive antinuclear antibody; Hematopoietic: thrombocytopenia; Immunologic:

anaphylaxis, Lupus-like syndrome, vasculitis; Integumentary: alopecia

The recommended dose for adults is $1200 \mathrm{mg}$ adminisMANAGEMENT OF OVERDOSE. While there has been no reported case of overdosage,

References: 1. Frick MH. Elo O. Haapa K, et al: Helsinki Heart Study. Primary prevention rial with gemfibrozil in middle-aged men with dyslipidemia. N EnglJ Med 1987:317:1237-1245 2. Manninen V, Elo O. Frick MH, et al: Lipid alterations and decline in the incider
coronary heart disease in the Helsinki Heart Study. JAMA 1988; $260: 641.651$ 3. Nikkila EA: Familial lipoprotein lipase deficiency and related disorders of chylomicron metabolism. In Stanbury J. B et al. (eds.): The Metabolic Basis of Inherited Disease, 5th ed. McGraw.Hill, 1983, Chap. 30, pp. 622-642

Caution - Federal law prohibits dispensing without prescription.

\section{PARKE-DAVIS}

Morris Plains, NJ 07950 
from donor pools preselected for a high antibody content against a specific antigen, eg, hepatitis B immune globulin (HBIG), varicella-zoster immune globulin, rabies immune globulin, and tetanus immune globulin. Like IG and IGIV, these preparations do not transmit infectious diseases.

Antitoxin: A solution of antibodies derived from the serum of animals immunized with specific antigens (eg, diphtheria antitoxin, botulinum antitoxin) used to achieve passive immunity or for treatment.

\section{Vaccination and immunization}

These terms are often used interchangeably. Vaccination and vaccine derive from vaccinia, the virus once used as smallpox vaccine. Thus, vaccination originally meant inoculation with vaccinia virus to render a person immune to smallpox. Although some persons still prefer that vaccination be restricted to this use, most use it to denote the administration of any vaccine or toxoid.

Immunization is a more inclusive term denoting the process of inducing or providing immunity artificially by administering an immunobiologic. Immunization can be active or passive.

Active immunization is the production of antibody or other immune responses to the administration of a vaccine or toxoid. Passive immunization means the provision of temporary immunity by the administration of preformed antibodies. Three types of immunobiologics are administered for passive immunization: (1) pooled human IG or IGIV, (2) spe- cific IG preparations, and (3) antitoxins.

Vaccination and immunization are used interchangeably in ACIP statements in reference to active immunization. Regardless of which term is used, administration of an immunobiologic cannot be automatically equated with the development of adequate immunity for a variety of reasons, many of which are discussed in the following information.

\section{Immunobiologics}

The specific nature and content of immunobiologics can differ. When immunobiologics against the same infectious agents are produced by different manufacturers, active and inert ingredients in the various products are not always the same. Practitioners are urged to become familiar with the constituents of the products they use.

\section{Suspending fluids}

These may be sterile water or saline or complex fluids containing small amounts of protein or other constituents derived from the medium or biologic system in which the vaccine is produced (eg, serum proteins, egg antigens, cellculture-derived antigens).

\section{Preservatives, stabilizers, and antibiotics}

These components of vaccines, antitoxins, and globulins are used to inhibit or prevent bacterial growth in viral cultures or the final product or to stabilize the antigens or antibodies. Allergic reactions can occur if the recipient is sensitive to one of these addi- tives (eg, mercurials, phenols, albumin, glycine).

\section{Adjuvants}

Many antigens evoke insufficient immunologic responses when given in their natural state. Efforts to enhance immunogenicity include mixing antigens with a variety of substances or adjuvants (eg, aluminum adjuvants such as aluminum phosphate).

\section{Route, site, and technique of immunization}

\section{Route}

Routes of administration are recommended for each immunobiologic (Table 1). To avoid unnecessary local or systemic effects and/ or to ensure optimal efficacy, the practitioner should not deviate from the recommended routes. Vaccines containing adjuvants must be injected deep into the muscle mass; they should not be administered subcutaneously or intradermally as they can cause local irritation, inflammation, granuloma formation, or necrosis.

\section{Site}

Injectable immunobiologics should be administered where there is little likelihood of local, neural, vascular, or tissue injury. Subcutaneous injections are usually administered into the thigh of infants and in the deltoid area of older children and adults. Intradermal injections are generally given on the volar surface of the forearm except for human diploid cell rabies vaccine with which reactions are less severe in the deltoid area. The preferred 
Table 2

Recommended Schedule for Active Immunization of Normal Infants and Children*

\begin{tabular}{|lll|}
$\begin{array}{c}\text { Recommended } \\
\text { age }^{\dagger}\end{array}$ & Vaccine(s) $\div$ & Comments \\
\hline 2 mo & DTP No. 1,\$ OPV No. 1\| & $\begin{array}{c}\text { OPV and DTP can be given } \\
\text { earlier in areas of high } \\
\text { endemicity }\end{array}$ \\
\hline mo & DTP No. 2, OPV No. 2 & $\begin{array}{l}\text { 6-wk to 2-mo interval desired } \\
\text { between OPV doses }\end{array}$ \\
& DTP No. 3 & $\begin{array}{l}\text { An additional dose of OPV } \\
\text { at this time is optional in } \\
\text { areas with a high risk of } \\
\text { poliovirus exposure }\end{array}$ \\
\hline
\end{tabular}

$\begin{array}{clc}15 \mathrm{mo} & \begin{array}{c}\text { MMR,\# DTP No. 4, } \\ \text { OPV No. } 3\end{array} & \begin{array}{c}\text { Completion of primary series } \\ \text { of DTP and OPV }\end{array} \\ 18 \mathrm{mo} & \mathrm{HbCV}^{* *} & \begin{array}{c}\text { Conjugate preferred over } \\ \text { polysaccharide }\end{array}\end{array}$

$\begin{array}{lll}4-6 \mathrm{yr} & \text { DTP No. } 5,+\neq \text { OPV No. } 4 & \text { At or before school entry } \\ 14-16 \mathrm{yr} & \text { Td\$\$ } & \begin{array}{c}\text { Repeat every } 10 \mathrm{yr} \\ \text { throughout life }\end{array}\end{array}$

* See Table 3 for the recommended immunization schedules for infants and children up to their seventh birthday not immunized at the recommended times.

* These recommended ages should not be construed as absolute, eg, 2 months can be 6 to 10 weeks. However, MMR should not be given to children $<12$ months of age. If exposure to measles disease is considered likely, then children 6 through 11 months old may be immunized with singleantigen measles vaccine. These children should be reimmunized with MMR when they are approximately 15 months of age.

$\doteqdot$ For all products used, consult the manufacturers' package enclosures for instructions regarding storage, handling, dosage, and administration. Immunobiologics prepared by different manufacturers can vary, and those of the same manufacturer can change from time to time. The package inserts are useful references for specific products, but they may not always be consistent with current ACIP and American Academy of Pediatrics immunization schedules.

$\$ \mathrm{DTP}=$ Diphtheria and tetanus toxoids and pertussis vaccine, adsorbed. DTP may be used up to the seventh birthday. The first dose can be given at 6 weeks of age and the second and third doses given 4 to 8 weeks after the preceding dose.

$\| \mathrm{OPV}=$ Poliovirus vaccine live oral, trivalent: contains poliovirus types 1,2 , and 3 .

- Provided at least 6 months have elapsed since DTP No. 3 or, if fewer than 3 doses of DTP have been received, at least 6 weeks since the last previous dose of DTP or OPV. MMR vaccine should not be delayed to allow simultaneous administration with DTP and OPV. Administering MMR at 15 months and DTP No. 4 and OPV No. 3 at 18 months continues to be an acceptable alternative. \# MMR $=$ Measles, mumps, and rubella virus vaccine, live. Counties that report $\geqslant 5$ cases of measles among preschool children during each of the last 5 years should implement a routine 2dose measles vaccination schedule for preschoolers. The first dose should be administered at 9 months or the first healthcare contact thereafter. Infants vaccinated before their first birthday should receive a second dose at about 15 months of age. Single-antigen measles vaccine should be used for children aged $<1$ year and MMR for children vaccinated on or after their first birthday. If resources do not allow a routine 2-dose schedule, an acceptable alternative is to lower the routine age for MMR vaccination to 12 months.

** $\mathrm{HbCV}=$ Vaccine composed of Haemophilus influenzae b polysaccharide antigen conjugated to a protein carrier. Children $<5$ years of age previously vaccinated with polysaccharide vaccine between the ages of 18 and 23 months should be revaccinated with a single dose of conjugate vaccine if at least 2 months have elapsed since the receipt of the polysaccharide vaccine.

†* If $\mathrm{HbCV}$ is not available, an acceptable alternative is to give Haemophilus influenzae b polysaccharide vaccine ( $\mathrm{HbPV}$ ) at age $\geqslant 24$ months. Children at high risk for Haemophilus influenzae type $\mathrm{b}$ disease where conjugate vaccine is not available may be vaccinated with $\mathrm{HbPV}$ at 18 months of age and revaccinated at 24 months.

$\div$ Up to the seventh birthday.

$\S \S \mathrm{Td}=$ Tetanus and diphtheria toxoids, adsorbed (for use in persons aged $\geqslant 7$ years): contains the same amount of tetanus toxoid as DTP or DT but a reduced dose of diphtheria toxoid. sites for intramuscular injections are the anterolateral aspect of the upper thigh and the deltoid muscle of the upper arm. In most infants, the anterolateral aspect of the thigh provides the largest muscle mass and is therefore the preferred site. An individual decision must be made for ech child based on the volume of the material to be administered and the size of the muscle into which it is to be injected. In adults, the deltoid is recommended for routine intramuscular vaccine administration, particularly for hepatitis B vaccine. The buttock should not be used routinely as a vaccination site for infants, children, or adults because of the risk of injury to the sciatic nerve. In addition, injection into the buttock has been associated with decreased immunogenicity of hepatitis B and rabies vaccines, presumably because of inadvertent subcutaneous injection or injection into deep fat tissue. If the buttock is used when very large volumes are to be injected or multiple doses are necessary (eg, large doses of IG), the central region should be avoided; only the upper, outer quadrant should be used.

\section{Techniques}

Syringes and needles used for injections must be sterile and preferably disposable to minimize the risk of contamination. For an intramuscular injection, the needle and syringe should be of sufficient length and bore to reach the muscle mass itself and prevent vaccine from seeping into subcutaneous tissue. For chil(continued on page 1203) 

THE EASY WAY TO ADDED CONTROL. Tenoretic

TENORMIN ${ }^{*}$ (atenolol) $50 \mathrm{mg}$ or $100 \mathrm{mg}$

and chlorthalidone $25 \mathrm{mg}$

Please consult complete product information before prescribing.
A summary follows:

TENORETIC (atenolol and chlorthalidone) is for the treatment of hypertension. It combines the antihypertensive activity of two agents: a beta, -selective (Casc

Inactive ingredients: magnesium stearate, microcrystalline cellulose, povidone, sodium starch glycolate.
INDICATONS AND USAGE. TENORETIC is indicated for the treatment of hypertension. This fxed-dose INDICATIONS AND USAGE: TENORETIC is indicated for the treatment of hypertension. This fixed-dose combination drug is not indicated for initial therapy of hypertension. If the fixed-dose combination represents the
dose approoriate to the individual patient's needs, it may be more convenient than the separate components. dose appropriate to the individual patient's needs, it may be more convenient than the separate components. than first degree, cardiogenic shock, overt cardiac fallure (see WARNINGS), anuria, hypersensitivity to this than first degree, cardiogenic shock, overt

product or to sultonamide-derived drugs.
WARNINGS: Cardiac Faliure: Sympathetic stimulation is necessary in supporting circulatory function in congestive heart failure, and beta blockade carries the potential hazard of further depressing myocardial contractility and precipitating more severe tailure. In hypertensive patients who have congestive heart failure controlled by digitalis and diuretics, TENORETIC should be administered cautiously. Both digitalis and atenolol slow AV conduction.

In Patients Without a History of Cardiac Failure: Continued depression of the myocardium with beta-blocking agents over a period of time can, in some cases, lead to cardiac failure. At the first sign or symptom of impending cardiac failure, patients receiving TENORETIC should be digitalized and/or be given additional diuretic therapy. Observe the patient closely. If cardiac falure continues despite adequat digitalization and diuretic therapy, TENORETIC therapy should be withdrawn.

Renal and Hepatic Disease and Electrolyte Disturbances: Since atenolol is excreted via the kidneys, TENORETIC should be used with caution in patients with impaired renal function.

In patients with renal disease, thiazides may precipitate azotemia. Since cumulative effects may develop in the presence of impaired renal function, if progressive renal impairment becomes evident, TENORETIC should ee discontinued.

In patients with impaired hepatic function or progressive liver disease, minor alterations in fluid and electrolyte balance may precipitate hepatic coma. TENORETIC should be used with caution in these patients

Ischemic Heart Disease: Although not yet reported with atenolol, following abrupt cessation of therapy with certain beta-blocking agents in patients with coronary artery disease, exacerbations of angina pectoris and, in some cases, myocardial infarction have been reported. Therefore, such patients should be cautioned against interruption of therapy without the physician's advice. Even in the absence of overt angina pectoris, when discontinuation of TENORETIC is planned, the patient should be carefully observed and should be advised to limit physical activity to a minimum. TENORETIC should be reinstated if withdrawal symptoms occur. Bronchospastic Diseases: PATIENTS WITH BRONCHOSPASTIC DISEASE SHOULD, IN GENERAL, NOT RECEIVE BETA BLOCKERS. Because of its relative beta,-selectivity, however, TENORETIC may be used with caution in patients with bronchospastic disease who do not respond to, or cannot tolerate, other antihypertensive treatment. Since beta,-selectivity is not absolute, the lowest possible dose of TENORETC should be used and a beta, -stimulating agent (bronchodilator) should be made available. If dosage must be increased, dividing the dose should be considered in order to achieve lower peak blood levels.

Anesthesia and Major Surgery: As with all beta-receptor blocking drugs, it may be decided to withdraw TENORETIC before surgery. In this case, 48 hours should be allowed to elapse between the last dose and anesthesia. If treatment is continued, ca

Beta blockers are compettive inhibitors of beta-receptor agonists and their effects on the heart can be reversed by administration of such agents: eg, dobutamine or isoproterenol with caution (see section on corrected with atropine (1.2 mg IV)

Metabolic and Endocrine Effects: TENORETIC may be used with caution in diabetic patients. Beta blockers may mask tachycardia occurring with hypoglycemia, but other manifestations such as dizziness and sweating may not be significantly affected. Atenolol does not potentiate insulin-induced hypoglycemia and, unlike nonselective beta blockers, does not delay recovery of blood glucose to normal levels.

Insulin requirements in diabetic patients may be increased, decreased, or unchanged; latent diabetes mellitus may become manifest during chlorthalidone administration.

Beta-adrenergic blockade may mask certain clinical signs (eg, tachycardia) of hyperthyroidism. Abrupt withdrawal of beta blockade might precipitate a thyroid storm; therefore, patients suspected of developing

thyrotoxicosis and from whom PENORETIC therapy is to be withdrawn should be monitored closely.

Because calcium excretion is decreased by thiazides, TENORETIC should be discontinued before carrying out tests for parathyroid function. Pathologic changes in the parathyroid glands, with hypercalcemia and hypophosphatemia, have been observed in a few patients on prolonged thiazide therapy; however, the common been seen.

Hyperuricemia may occur or acute gout may be precipitated in certain patients receiving thiazide therapy. PRECAUTIONS: General: Electrolyte and Fluid Balance Status: Periodic determination of serum electrolytes o detect possible electrolyte imbalance should be performed at appropriate intervals. Patients should be observed for clinical signs of fluid or electrolyte imbalance, ie, hyponatremia, hypochloremic
alkalosis, and hypokalemia. Serum and urine electrolyte determinations are particularly important when the patient is vomiting excessively or receiving parenteral fluids. Warning signs or symptoms of fluid and electrolyte imbalance include dryness and vomiting.

Hypokalemia may develop, especially with brisk diuresis, when severe cirrhosis is present, or during concomitant use of corticosteroids or ACTH. Interference with adequate oral electrolyte intake will also contribute to hypokalemia. Hypokalemia can
sensitize or exaggerate the response of the heart to the toxic effects of digitalis (eg, increased ventricular sensitize or exaggerate the response of the heart to the toxic effects of digitalis (eg, increased ventricular
irritability). Hypokalemia may be avoided or treated by use of potassium supplements or foods with a high potassium content.

Any chloride deficit during thiazide therapy is generally mild and usually does not require specific treatment except under extraordinary circumstances (as in liver disease or renal disease). Dilutional hyponatremia may occur in edematous patients in hot weather; appropriate therapy is water restriction rather than administration
of salt except in rare instances when the hyponatremia is life threatening. In actual salt depletion, appropriate replacement is the therapy of choice.

Drug Interactions: TENORETIC may potentiate the action of other antihypertensive agents used
Deplacement is the therapy of choice. Drug Interactions: TENORETIC may potentiate the action of other antihypertensive agents used
concomitantly. Patients treated with TENORETIC plus a catecholamine depletor (eg, reserpine) should be closely observed for evidence of hypotension and/or marked bradycardia which may produce vertigo, syncope, or postural hypotension.

Thiazides may decrease arterial responsiveness to norepinephrine. This diminution is not sufficient to preclude the therapeutic effectiveness of norepinephrine. Thiazides may increase the responsiveness to tubocurarine.
Lithium generally should not be given with diuretics because they reduce its renal clearance and add a high Lithium generally should not be given with diuretics because they reduce its renal clearance and add a high
risk of lithium toxicity. Read circulars for lithium preparations before use of such preparations with TENOREFIC. Should it be decided to discontinue therapy in patients receiving TENORETIC and clonidine concurrently, the TENORETIC should be discontinued several days before the gradual withdrawal of clonidine.

Other Precautions: In patients receiving thiazides, sensitivity reactions may occur with or without a history of allergy or bronchial asthma. The possible exacerbation or activation of systemic lupus erythematosus has been
reported. The anthypertensive effects of thiazides may be enhanced in the postsympathectomy patient.
Carcinogenesis, Mutagenesis, Impairment of Fertility: Two long-term (maximum dosing duration of 18 24 months) rat studies and one long-term (maximum dosing duration of 18 months) mouse study with atenolo each employing dose levels as high as $300 \mathrm{mg} / \mathrm{kg} /$ day or 150 times the maximum recommended human dose did not indicate a carcinogenic potential in rodents.

Atenolol was negative in the mouse dominant lethal test, the Chinese hamster in vivo cytogenetic test, and the Salmonella typhimurium back mutation test (Ames test), with or without metabolic activation.

Fertility of male or female rats (evaluated at dose levels as high as $200 \mathrm{mg} / \mathrm{kg} /$ day or 100 times the maxim recommended human dose) was unaffected by atenolol administration.

Animal Toxicology: Six-month oral studies were conducted in rats and dogs using TENORETIC dosages to $12.5 \mathrm{mg} / \mathrm{kg}$ day (approximately 5 times the proposed maximum therapeutic dose). There were no function or morphological abnormalities resulting from dosing either compound alone or together other than minor changes in heart rate, blood pressure, and urine chemistry, which were attributed to the known

pharmacologic properties of atenolol and/or chlorthalidone.

Chronic studies of atenolol performed in animals have revealed the occurrence of vacuolation of epithelial cells of Brunner's glands in the duodenum of both male and female dogs at all tested dose levels (starting at $15 \mathrm{mg} / \mathrm{kg}$ day or 7.5 times the maximum recommended human dose) and increased incidence of atrial degeneration of hearts of male rats at 300

commended human dose, respectively.

Use in Pregnancy: Pregnancy Category C. TENORETIC (atenolol and chlorthalidone) was studied for teratogenic potential in the rat and rabbit. Doses of 10,100 , and $300 \mathrm{mg} / \mathrm{kg} /$ day were administered orally to pregnant rats, with no teratologic effects observed. Two studies were conducted in rabbits. In the first study, pregnant rabbits were dosed with 10,100 or $200 \mathrm{mg} / \mathrm{kg}$ day. No teratologic changes were noted; embryonic resorptions were observed at all dose levels (ranging from approximately 5 times to 100 times the maximum recommended human dose). In a second raboit study, dosages were 5,10 , and $25 \mathrm{mg} / \mathrm{kg} /$ day. No teratogen or embryotoxic effects were demonstrated. It is concluded that the no-eftect level for embryonic resorptions is
$25 \mathrm{mg} / \mathrm{kg} / \mathrm{day}$ (approximately 12.5 times the maximum recommended human dose) or greater. TENORETIC should be used during pregnancy only if the potential benefit justifies the potential risk to the fetus.

Atenole- - Atenolol has been shown to produce a dose-related increase in embryolfetal resorptions in rats doses equal to or greater than $50 \mathrm{mg} / \mathrm{kg}$ or 25 or more times the maximum recommended human dose.

$25 \mathrm{mg} k \mathrm{~kg}$ or 12.5 times the maximum in rabbits, the compound was not evaluated in rabbits at doses above studies in pregnant women.

Chlorthalidene-Thiazides cross the placental barrier and appear in cord blood. The use of chlorthalidone and related drugs in pregnant women requires that the anticipated benefits of the drug be weighed against possible hazards to the fetus. These hazards include feta

Nursing Mothers: It is not established to what extent this drug is excreted in human milk. Since most drug are excreted in human milk, nursing should not be undertaken by mothers receiving TENORETIC.

are excreted in human milk, nursing should not be undertaken by mothers receiving
Pediatric Use: Safety and effectiveness in children have not been established

Pediatric Use: Safety and effectiveness in children have not been established.
ADVERSE REACTIONS: TENORETIC is usually well tolerated in properly selected patients. Most adverse effects have been mild and transient. The adverse effects observed for TENORETIC are essentially the same as those seen with the individual components.

Atenolol: The frequency estimates that follow derive from controlled studies in which adverse reactions were either volunteered by the patient (US studies) or elicited, eg, by checklist (foreign studies). The reported Irequency of elicited adverse effects was higher for both atenolol and placebo-treated patients than when thes

reactions were volunteered. Where

The data present these estimates in terms of percentages: first from the US studies (volunteered side effect and then from both US and foreign studies (volun
US STUDIES (\% ATENOLOL.\% PLACEBO):

CARDIOVASCULAR: bradycardia $(3 \%-0 \%)$, cold extremities $(0 \%-0.5 \%)$, postural hypotension $(2 \%-1 \%)$, eg pain $10 \% \cdot 0.5 \%$

CENTRAL NERVOUS SYSTEMNEUROMUSCULAR: dizziness ( $4 \%-1 \%)$, vertigo $(2 \%-0.5 \%)$, lightdepression $(0.6 \% \cdot 0.5 \%)$, dreaming $(0 \%-0 \%)$

GASTROINTESTINAL: diarrhea $(2 \%-0 \%)$, nausea $(4 \%-1 \%)$

RESPIRATORY (See WAANINGS): wheeziness $(0 \%-0 \%)$, dyspnea $(0.6 \%-1 \%)$

CARDIOVASCULAR: bradycardia $(3 \%-0 \%)$, cold extremities (12\%-5\%), postural hypotension ( $4 \%-5 \%)$, eg pain $(3 \%-1 \%)$

headedness $(3 \%-0.7 \%)$, tiredness $(26 \% \cdot 13 \%)$, fatigue $(6 \%-5 \%)$, lethargy $(3 \%-0.7 \%)$, drowsiness $(2 \%-0.5 \%)$, depression (12\%-9\%), dreaming $(3 \%-1 \%)$

GASTROINTESTINAL: diarnhea $(3 \%-2 \%)$, nausea $(3 \%-1 \%)$

RESPIRATORY (see WARNINGS): wheeziness (3\%-3\%), dyspnea (6\%-4\%)

Miscellaneous: There have been reports of skin rashes and or dry eyes associated with the use of beta-adrenergic blocking drugs. The reported incidence is small, and in most cases, the symptoms have clea when treatment was withdrawn. Discontinuance of the drug should be considered if any such reaction is not otherwise explicable. Patients should be closely monitored following cessation of therapy.

Chlorthalidone: Cardiovascular: orthostatic hypotension; Gastrointestinal: anorexia, gastric irritation, vomitin cramping, constipation, jaundice (intrahepatic cholestatic jaundice), pancreatitis; CNS: vertigo, paresthesias, xanthopsia; Hematologic: leukopenia, agranulocytosis, thrombocytopenia, aplastic anemia; Hypersensitivity: purpura, photosensitivity, rash, urticaria, necrotizing angitits (vasculitis, cutaneous vasculitis), Lyell's syndrome (toxic epidermal necrolysis); Miscellaneous: hyperglycemia, glycosuria, hyperuricemia, muscle spasm, weakness, restlessness. Clinical trials of TENORE TIC conducted in the United States (89 patients treated wit PNORETC) revealed no new or unexpected adverse effects.

Potential Adverse Effects: In addition, a variety of adverse effects not observed in clinical trials with aten but reported with other beta-adrenergic blocking agents, should be considered potential adverse effects of atenolol. Nervous System: reversible mental depression progressing to catatonia; hallucinations; an acute reversible syndrome characterized by disorientation for time and place, short-term memory loss, emotional lability, slightly clouded sensorium, and decreased pertormance on neuropsychometrics; Cardiovascular: intensification of AV block (see CONTRAINDICATIONS); Gastrointestinal: mesenteric arterial thrombosis, ischemic colitis; Hematologic: agranulocytosis, nonthrombocytopenic purpura, thrombocytopenic purpura Allergic: erythematous rash, fever combined with aching and sore throat, laryngospasm and respiratory distress; Miscellaneous: reversible alopecia Peyronie's disease.

There have been reports of a syndrome comprising psoriasiform skin rash, coniunctivitis sicca, otitis, and sclerosing serositis attributed to the beta-adrenergic receptor blocking agent, practolol. This syndrome has not been reported with TENORETIC or TENORMIN (atenolo

Clinical Laboratory Test Findings: Clinically important changes in standard laboratory parameters were rarely associated with the administration of TENORETIC. The changes in laboratory parameters were not progressive and usually were not associated with clinical manifestations. The most common changes were increases in uric acid and decreases in serum potassium.

DOSAGE AND ADMINISTRATION: Initial dose should be one TENORETIC 50 tablet once a day, If optim response is not achieved, the dosage should be increased to one TENORETIC 100 tablet once a day. Package insert should be consulted for dosage adjustments in cases of severe impairment of renal function. white, round, biconvex, uncoated tablets with $\mathrm{ICl}$ on one side and 115 on the other side, bisected) are supplied in bottles of 100 tablets.

TENORETIC 100 Tablets (atenolol $100 \mathrm{mg}$ and chlorthalidone $25 \mathrm{mg}$ ), NDC 0310-0117 (white, round, biconvex, uncoated tablets with ICl on one side and 117 on the other side) are supplied in bottles of 100 tab Protect from heat, light, and moisture. Dispense in well-closed light-resistant container.

A12/86

References: 1. Curry RC Jr: Dose ranging and satety evaluation of atenolol administered once daily to mild to moderate essential hypertensives, Study HPA 7-237-5-1. Data on file, ICI Pharma, Wilmington, Delaware. 2. Nash DT: Single-blind dose determination study in mild hypertensives using fixed rising Delaware. 3. Petrie JC, Jeffers TA, Webster J: Atenolol once daily in mild hypertension, Study 40. Data on file, ICI Pharma, Wilmington, Delaware. 4. Russell JG, Mayhew SR, Humphries IS: Chlorthalidone in mild hypertension-dose response relationship. Eur J Clin Pharmacol 1981;20:407-411. 
dren, a 20- or 22-gauge needle 1 to $1^{1 / 4}$ inches long is recommended. For small infants, a 25gauge $5 / 8$-inch-long needle may be adequate. For adults, the suggested needle length is $1^{1 / 2}$ inches. For subcutaneous or intradermal injections, a 25-gauge needle $5 / 8$ to $3 / 4$ inches long is recommended.

Before the injection is given, the needle is inserted in the site and the syringe plunger pulled back; if blood appears, the needle should be withdrawn and a new site selected. The process should be repeated until no blood appears. A separate needle and syringe should be used for each vaccine injected. Disposable needles and syringes should be discarded into labeled, punctureproof containers to prevent accidental needlesticks or reuse. If more than one vaccine preparation is administered or if vaccine and IG are administered simultaneously, each should be given at a different site.

\section{Dosage}

The recommendations on dosages of immunobiologics are derived from theoretical considerations, experimental trials, and clinical experience. Administration of volumes smaller than those recommended, such as split doses or intradermal administration (unless specifically recommended), can result in inadequate protection. Use of larger than the recommended dose can be hazardous because of excessive local or systemic concentrations of antigens.
The ACIP strongly discourages any variation from the recommended volume or number of doses of any vaccine. Some practitioners use smaller, divided, doses of vaccine, thereby reducing the total immunizing dose. Others use multiple smaller doses that together equal a full immunizing dose (eg, diphtheria and tetanus toxoids and pertussis vaccine [DTP]) in an effort to reduce reactions. However, the serologic response, clinical efficacy, and/or frequency and severity of adverse reactions of such schedules have not been adequately studied.

\section{Age at which immunobiolo- gics are administered}

Several factors influence recommendations concerning the age at which vaccines are administered (Table 2); they are agespecific risks of disease, age-specific risks of complications, ability of persons of a given age to respond to the vaccine(s), and potential interference with the immune response by passively transferred maternal antibody. In general, vaccines are recommended for the youngest age group at risk whose members are known to develop an acceptable antibody response to vaccination.

\section{Spacing of immunobiologics}

\section{Multiple doses of same antigen} Some products require administration of more than one dose for development of an adequate antibody response. In addition, some products require periodic re- inforcement (booster) doses to maintain protection. In recommending the ages and/or intervals for multiple doses, the ACIP takes into account risks from disease and the need to induce or maintain satisfactory protection (Tables 2,3, and 4).

Intervals between doses that are longer than those recommended do not lead to a reduction in final antibody levels. Therefore, it is not necessary to restart an interrupted series of an immunobiologic or to add extra doses.

In contrast, giving doses of a vaccine or toxoid at less than recommended intervals may lessen the antibody response and therefore should be avoided. Doses given at less than recommended intervals should not be counted as part of a primary series.

Some vaccines produce local or systemic symptoms in certain recipients when given too frequently (eg, Td, DT, and rabies). Such reactions are thought to result from the formation of antigen-antibody complexes. Good recordkeeping, careful patient histories, and adherence to recommended schedules can decrease the incidence of such reactions without sacrificing immunity.

\section{Different antigens}

Experimental evidence and extensive clinical experience have strengthened the scientific basis for giving certain vaccines at the same time. Many of the widely used vaccines can safely and effectively be given simultaneously (ie, on the same day, not at the same site). This knowledge is 
Table 3

Recommended Immunization Schedule for Infants and Children up to the Seventh Birthday Not Immunized at the Recommended Time in Early Infancy*

(See individual recommendations for details)

\begin{tabular}{|c|c|c|}
\hline Timing & Vaccine & Comments \\
\hline First visit & $\begin{array}{l}\text { DTP No. } 1, \div \text { OPV No. } 1, \div \\
\text { MMR if child is } \geqslant 15 \text { mo } \\
\text { and HbCV } \| \text { if child is } \\
\text { aged } \geqslant 18 \text { mo }\end{array}$ & $\begin{array}{l}\text { DTP, OPV, and MMR should } \\
\text { be administered simulta- } \\
\text { neously to children aged } \\
\geqslant 15 \mathrm{mo} \text {, if appropriate. } \\
\text { DTP, OPV, MMR, and HbCV } \\
\text { may be given simultaneously } \\
\text { to children } 18 \text { mo to } 5 \mathrm{yr}\end{array}$ \\
\hline
\end{tabular}

2 mo after DTP No. DTP No. 2,9 OPV No. 2
1, OPV No. 1

2 mo after DTP No. 2 DTP No. 39

An additional dose of OPV at this time is optional in areas with a high risk of poliovirus exposure

6-12 mo after DTP DTP No. 4 , OPV No. 3
No. 3

Preschool \# (4-6 yr) DTP No. 5, OPV No. 4

Preferably at or before school entry

$15-16$ yr

$\mathrm{Td}^{* *}$

Repeat every $10 \mathrm{yr}$ throughout life

*If initiated in the first year of life, give DTP No. 1, 2, and 3 and OPV No. 1 and 2 according to this schedule; give MMR when the child becomes 15 months old

$\div$ DTP $=$ Diphtheria and tetanus toxoids and pertussis vaccine, adsorbed. DTP can be used up to the seventh birthday.

$\$ \mathrm{OPV}=$ Poliovirus vaccine live oral, trivalent; contains poliovirus types 1,2 , and 3 .

$\$ \mathrm{MMR}=$ Measles, mumps, and rubella virus vaccine, live (see text for discussion of single vaccines versus combination).

$\| \mathrm{HbCV}=$ Vaccine composed of Haemophilus influenzae $\mathrm{b}$ polysaccharide antigen conjugated to a protein carrier. If $\mathrm{HbCV}$ is not available, an acceptable alternative is to give Haemophilus influenzae b polysaccharide vaccine ( $\mathrm{HbPV}$ ) at 24 months of age. If $\mathrm{HbCV}$ is unavailable and if the child is at high risk for Haemophilus influenzae type b disease, $\mathrm{HbPV}$ may be given at 18 months of age with a second dose at 24 months. Children aged $<5$ years who were previously vaccinated with $\mathrm{HbPV}$ between 18 and 23 months of age should be revaccinated with a single dose of $\mathrm{HbCV}$ at least 2 months after the initial dose of $\mathrm{HbPV}$. Either $\mathrm{HbCV}$ or $\mathrm{HbPV}$ can be administered up to the fifth birthday. However, they are not generally recommended for persons $\geqslant 5$ years of age.

- The second and third doses of DTP can be given 4 to 8 weeks after the preceding dose.

\# The preschool doses are not necessary if the fourth dose of DTP and third dose of OPV are administered after the fourth birthday.

** Td $=$ Tetanus and diphtheria toxoids, adsorbed (for use in persons aged $\geqslant 7$ years): contains the same dose of tetanus toxoid as DTP or DT and a reduced dose of diphtheria toxoid.

particularly helpful when there is imminent exposure to several infectious diseases, preparation for foreign travel, or uncertainty that the person will return for further doses of vaccine.
1. Simultaneous administration-In general, inactivated vaccines can be administered simultaneously at separate sites. However, when vaccines commonly associated with local or systemic side effects (eg, cholera, typhoid, and plague) are given simultaneously, the side effects can be accentuated. Whenever possible, these vaccines should be given on separate occasions.

Simultaneous administration of pneumococcal polysaccharide vaccine and whole-virus influenza vaccine elicits satisfactory antibody responses without increasing the incidence or severity of adverse reactions. Simultaneous administration of the pneumococcal vaccine and splitvirus influenza vaccine can also be expected to yield satisfactory results. Influenza vaccine should be administered annually to the target population.

In general, simultaneous administration of the most widely used live and inactivated vaccines has not resulted in impaired antibody responses or increased rates of adverse reactions. Administration of combined measles, mumps, and rubella (MMR) vaccine yields results similar to administration of individual measles, mumps, and rubella vaccines at different sites. Therefore, there is no medical basis for giving these vaccines separately for routine immunization instead of the preferred MMR combined vaccine.

There are equivalent antibody responses and no clinically significant increases in the frequency of adverse events when 
DTP, MMR, and oral polio vaccine $(\mathrm{OPV})$ or inactivated polio vaccine (IPV) are administered either simultaneously at different sites or separately. As a result, routine simultaneous administration of MMR, DTP, and OPV (or IPV) to all children $\geqslant 15$ months who are eligible to receive these vaccines is recommended. Administration of MMR at 15 months followed by DTP and OPV (or IPV) at 18 months remains an acceptable alternative, especially for children with caregivers known to be generally compliant with other healthcare recommendations. Data are lacking on concomitant administration of Haemophilus influenzae b conjugate vaccine ( $\mathrm{HbCV}$ ) or Haemophilus influenzae b polysaccharide vaccine (HbPV) and MMR and OPV vaccine. If the child might not be brought back for future immunizations, the simultaneous administration of all vaccines (including DTP, OPV, $\mathrm{MMR}$, and $\mathrm{HbCV}$ or $\mathrm{HbPV}$ ) appropriate to the age and previous vaccination status of the recipient is recommended. Hepatitis B vaccine given with DTP and OPV or given with yellow fever vaccine is as safe and efficacious as these vaccines administered separately.

The antibody responses of both cholera and yellow fever vaccines are decreased if given simultaneously or within a short time of each other. If possible, cholera and yellow fever vaccinations should be separated by at least 3 weeks. If there are time constraints and both vaccines are necessary, the injections can be
Table 4

Recommended Immunization Schedule for Persons $\geqslant 7$ Years of Age Not Immunized at the Recommended Time in Early Infancy (See individual ACIP recommendations for details)

\begin{tabular}{|c|c|c|}
\hline Timing & Vaccines & Comments \\
\hline First visit & $\begin{array}{l}\text { Td No. } 1, * \text { OPV No. } 1, \dagger \\
\text { and MMR } \stackrel{\dagger}{\leftarrow}\end{array}$ & $\begin{array}{l}\text { OPV not routinely recom- } \\
\text { mended for persons aged } \\
\geqslant 18 \mathrm{yr}\end{array}$ \\
\hline $\begin{array}{l}2 \text { mo after Td No. } 1 \text {, } \\
\text { OPV No. } 1\end{array}$ & Td No. 2 , OPV No. 2 & $\begin{array}{l}\text { OPV may be given as soon as } \\
6 \mathrm{wk} \text { after OPV No. } 1\end{array}$ \\
\hline $\begin{array}{l}\text { 6-12 mo after Td } \\
\text { No. } 2 \text {, OPV No. } 2\end{array}$ & Td No. 3 , OPV No. 3 & $\begin{array}{l}\text { OPV No. } 3 \text { may be given as } \\
\text { soon as } 6 \text { wk after OPV } \\
\text { No. } 2\end{array}$ \\
\hline $10 \mathrm{yr}$ after Td No. 3 & Td & $\begin{array}{l}\text { Repeat every } 10 \mathrm{yr} \\
\text { throughout life }\end{array}$ \\
\hline \multicolumn{3}{|c|}{ 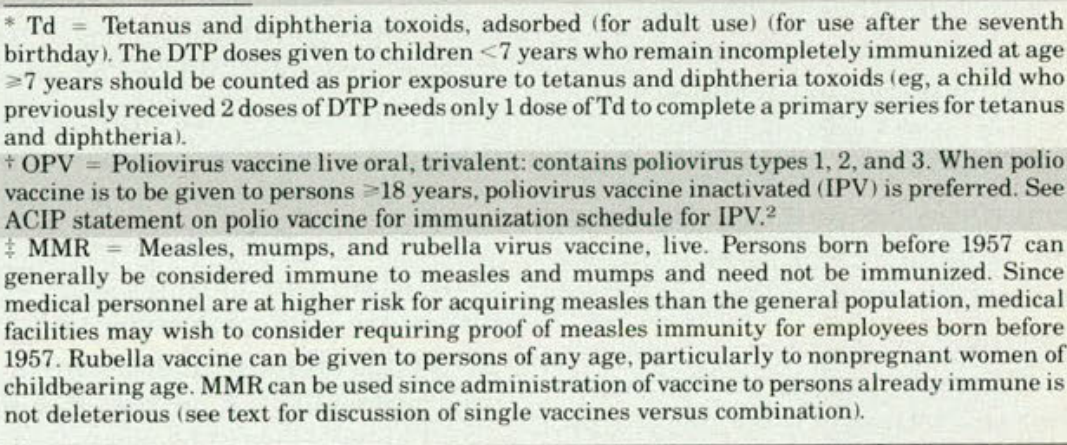 } \\
\hline
\end{tabular}

Table 5

Guidelines for Spacing Live and Killed Antigen Administration

Antigen combination Recommended minimum interval between doses

$\geqslant 2$ Killed antigens None; may be given simultaneously or at any interval between doses*

Killed and live antigens None; may be given simultaneously or at any interval between dosest

$\geqslant 2$ Live antigens

4-wk minimum interval if not administered simultaneously

* If possible, vaccines associated with local or systemic side effects (eg, cholera, typhoid, plague vaccines) should be given on separate occasions to avoid accentuated reactions.

- Cholera vaccine with yellow fever vaccine is the exception. If time permits, these antigens should not be administered simultaneously, and at least 3 weeks should elapse between administration of yellow fever vaccine and cholera vaccine. If the vaccines must be given simultaneously or within 3 weeks of each other, the antibody response may not be optimal 
given simultaneously or within a 3-week period with the understanding that antibody response may not be optimal. Decisions on the need for yellow fever and cholera immunizations should take into account the amount of protection afforded by the vaccine, the possibility that environmental or hygienic practices may be sufficient to avoid disease exposure, and the existence of vaccination requirements for entry into a country.

2. Nonsimultaneous administration-Inactivated vaccines do not interfere with the immune response to other inactivated vaccines or to live vaccines except, as noted above, with cholera and yellow fever vaccines. In general, an inactivated vaccine can be given either simultaneously or at any time before or after a different inactivated vaccine or a live vaccine.

There are theoretical concerns that the immune response to one live-virus vaccine might be impaired if given within 30 days of another. Whenever possible, livevirus vaccines not administered on the same day should be given at least 30 days apart (Table 5). Live virus vaccines can interfere with the response to a tuberculin test.

Tuberculin testing can be done either on the same day that livevirus vaccines are administered or 4 to 6 weeks afterwards.

\section{Immune globulin}

If administration of an IG preparation becomes necessary because of imminent exposure to disease, live-virus vaccines can be given simultaneously with the
IG product, with the recognition that vaccine-induced immunity might be compromised. The vaccine should be administered at a site remote from that chosen for the IG inoculation. Vaccination should be repeated about 3 months later unless serologic testing indicates that specific antibodies have been produced. OPV and yellow fever vaccines are exceptions, however, and are not affected by administration of IG at any time.

Live, attenuated vaccine viruses might not replicate successfully, and antibody response could be diminished when the vaccine is given after IG or specific IG preparations. Whole blood or other antibody-containing blood products can interfere with the antibody response to measles, mumps, and rubella vaccines. In general, these parenterally administered live vaccines should not be given for at least 6 weeks, and preferably 3 months, after IG administration. However, the postpartum vaccination of susceptible women with rubella vaccine should not be delayed because of receipt of anti-Rho(D) IG (human) or any other blood product during the last trimester of pregnancy or at delivery. These women should be vaccinated immediately after delivery and, if possible, tested in 3 months to ensure that rubella immunity was established.

If administration of IG preparations becomes necessary after a live-virus vaccine has been given, interference can occur. Usually, vaccine virus replication and stimulation of immunity will occur 1 to 2 weeks after vacci- nation. Thus, if the interval between administration of live-virus vaccine and subsequent administration of an IG preparation is $<14$ days, vaccination should be repeated at least 3 months after the IG product was given, unless serologic testing indicates that antibodies were produced.

In general, there is little interaction between IG preparations and inactivated vaccines. Therefore, inactivated vaccines can be given simultaneously or at any time before or after an IG product is used. For example, postexposure prophylaxis with simultaneously administered hepatitis $\mathrm{B}$, rabies, or tetanus IG and the corresponding inactivated vaccine or toxoid does not impair the immune response and provides immediate protection and longlasting immunity. The vaccine and IG should be given at different sites, and standard doses of the corresponding vaccine should be used. Increasing the vaccine dose volume or number of immunizations is not indicated (Table 6).

\section{Hypersensitivity to vaccine components}

Vaccine components can cause allergic reactions in some recipients. These reactions can be local or systemic, including mild to severe anaphylaxis (eg, hives, swelling of the mouth and throat, difficulty breathing, hypotension, or shock). The responsible vaccine components can derive from: (1) animal protein, (2) antibiotics, (3) preservatives, and (4) stabilizers. The most common animal protein allergen is egg pro- 


\section{federal update

tein found in vaccines prepared using embryonated chicken eggs or chicken embryo cell cultures (eg, yellow fever, mumps, measles, and influenza vaccines). Ordinarily, persons who are able to eat eggs or egg products safely can receive these vaccines; persons with histories of anaphylactic allergy to eggs or egg proteins should not.

Asking persons whether they can eat eggs without adverse effects is a reasonable way to screen for those who might be at risk from receiving measles, mumps, yellow fever, and influenza vaccines. Protocols requiring extreme caution have been developed for testing and vaccinating with measles and mumps vaccines those persons with anaphylactic reactions to egg ingestion. ${ }^{4}$ A regimen for administering influenza vaccine to children with egg hypersensitivity and severe asthma has also been developed. ${ }^{5}$

Rubella vaccine is grown in human diploid cell cultures and can safely be given to persons with histories of severe allergy to eggs or egg proteins.

Some vaccines contain trace amounts of antibiotics to which patients may be hypersensitive. The information provided in the vaccine package insert should be carefully reviewed before a decision is made whether the rare patient with such hypersensitivity should be given the vaccine(s). No currently recommended vaccine contains penicillin or its derivatives.

MMR and its individual component vaccines contain trace amounts of neomycin. Although the amount present is less than

Table 6

Guidelines for Spacing and Administration of Immune Globulin (IG) Preparations and Vaccines

\section{Simultaneous administration: Immunobiologic combination}

IG and killed antigen

IG and live antigen

Nonsimultaneous administration:

Immunobiologic administered

\begin{tabular}{|c|c|c|}
\hline First & Second & $\begin{array}{l}\text { Recommended minimum interval } \\
\text { between doses }\end{array}$ \\
\hline IG & Killed antigen & None \\
\hline Killed antigen & IG & None \\
\hline IG & Live antigen & $6 \mathrm{wk}$ and preferably $3 \mathrm{mo}^{*}$ \\
\hline Live antigen & IG & $2 \mathrm{wk}$ \\
\hline
\end{tabular}

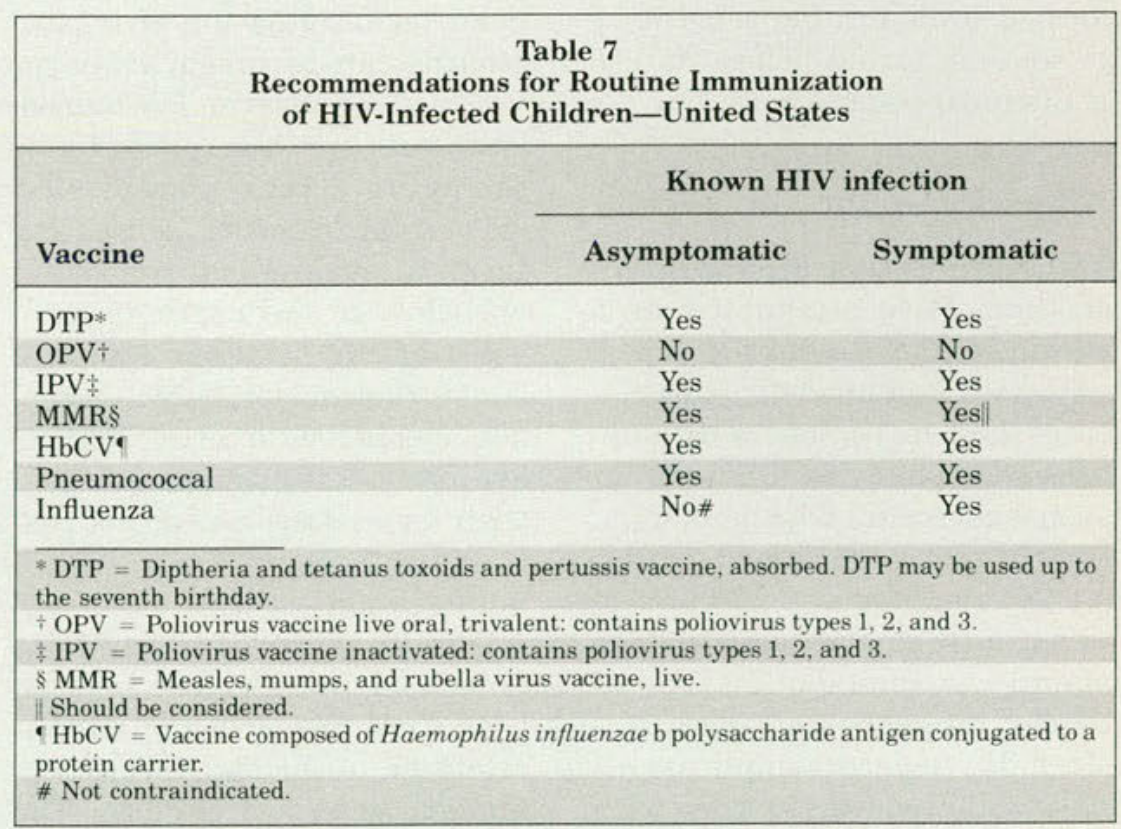


would usually be used for the skin test to determine hypersensitivity, persons who have experienced anaphylactic reactions to neomycin should not be given these vaccines. Most often, neomycin allergy is a contact dermatitis, a manifestation of a delayedtype (cell-mediated) immune response rather than anaphylaxis. A history of delayed-type reactions to neomycin is not a contraindication for these vaccines.

Bacterial vaccines, such as cholera, DTP, plague, and typhoid, are frequently associated with local or systemic adverse effects, such as redness, soreness, and fever. These reactions are difficult to link with a specific sensitivity to vaccine components and appear to be toxic rather than hypersensitive. On rare occasions, urticarial or anaphylactic reactions in DTP, DT, or Td recipients have been reported. When such events are reported, appropriate skin tests should be performed to determine sensitivity to tetanus toxoid before its use is discontinued. ${ }^{6}$

\section{Altered immunocompetence}

Virus replication after administration of live, attenuated-virus vaccines can be enhanced in persons with immunodeficiency diseases and in persons with suppressed capacity for immune response as occurs with leukemia, lymphoma, generalized malignancy, symptomatic HIV infections, or therapy with alkylating agents, antimetabolites, radiation, or large amount's of corticosteroids. Severe complications have followed vaccination with live, attenuated-virus vaccines and with live-bacteria vaccines (eg, BCG) in patients with leukemia, lymphoma, or suppressed immune responses. In general, these patients should not be given live vaccines, with the exceptions noted below.

If polio immunization is indicated for immunosuppressed patients, their household members, or other close contacts, these persons should be given IPV rather than OPV. Although a protective immune response cannot be assured in the immunocompromised patient, some protection may be provided. Because of the possibility of immunodeficiency in other children born to a family in which one such case has occurred, no family members should receive OPV unless the immune statuses of the intended recipient and all other children in the family are known.

Patients with leukemia in remission whose chemotherapy has been terminated for at least 3 months can be given live-virus vaccines. Short-term, low-to-moderate dose systemic corticosteroid therapy ( $<2$ weeks), topical steroid therapy (eg, nasal, skin), longterm alternate-day treatment with low to moderate doses of short-acting systemic steroids, and intra-articular, bursal, or tendon injection with corticosteroids are not immunosuppressive in their usual doses and do not contraindicate live-virus vaccine administration.

The growing number of infants and preschoolers infected with HIV has directed special attention to the appropriate immunization of such children. The evaluation and testing for HIV infection of asymptomatic children presenting for vaccines is not necessary before decisions concerning immunization are made. The inactivated childhood vaccines (eg, DTP or HbCV) should be given to HIV-infected children regardless of whether HIV symptoms are present. Although OPV has not been harmful when administered to asymptomatic HIVinfected children, IPV is the vaccine of choice if the child is known to be infected. The use of IPV not only eliminates any theoretical risk to the vaccinee but also prevents the possibility of vaccine virus spread to immunocompromised close contacts. Asymptomatically infected persons in need of MMR should receive it. Also, MMR should be considered for all symptomatic HIVinfected children because measles disease can be severe in symptomatic HIV-infected children. Limited studies of MMR immunization in both asymptomatic and symptomatic HIV-infected patients have not documented serious or unusual adverse events. In addition, pneumococcal vaccine is recommended for any child infected with HIV. Influenza vaccine is recommended for children with symptoms of HIV infection (Table 7).

\section{Febrile illness}

The decision to administer or delay vaccination because of a current or recent febrile illness depends largely on the severity of symptoms and on the etiology of the disease.

Although a moderate or severe 
febrile illness is reason to postpone immunizations, minor illnesses such as mild upper-respiratory infections (URI) with or without low-grade fever are not contraindications for vaccination. In persons whose compliance with medical care cannot be assured, it is particularly important to take every opportunity to provide appropriate vaccinations.

Children with moderate or severe febrile illnesses can be vaccinated as soon as the child has recovered. This precaution to wait avoids superimposing adverse effects of the vaccine on the underlying illness or mistakenly attributing a manifestation of the underlying illness to the vaccine.

Routine physical examinations or measurements of temperatures are not prerequisites for vaccinating infants and children who appear to be in good health. Asking the parent or guardian if the child is ill, postponing vaccination in those with moderate or severe febrile illnesses, and immunizing those without contraindications to vaccination are appropriate procedures in childhood immunization programs.

\section{Vaccination during preg- nancy}

Because of a theoretical risk to the developing fetus, pregnant women or women likely to become pregnant within 3 months after vaccination should not be given live, attenuated-virus vaccines. With some of these vaccines - particularly rubella, mea- sles, and mumps-pregnancy is a contraindication. Both yellow fever vaccine and OPV, however, can be given to pregnant women who are at substantial risk of exposure to natural infection. When a vaccine is to be given during pregnancy, waiting until the second or third trimester is a reasonable precaution to minimize concern over teratogenicity. Although there are theoretical risks, there is no evidence of congenital rubella syndrome in infants born to susceptible mothers who inadvertently were given rubella vaccine during pregnancy.

Persons given measles, mumps, or rubella vaccines can shed but not transmit these viruses. These vaccines can be administered safely to the children of pregnant women. Although live polio virus is shed by persons recently immunized with OPV (particularly after the first dose), this vaccine can also be administered to the children of pregnant women because experience has not revealed any risk of polio vaccine virus to the fetus.

There is no convincing evidence of risk to the fetus from immunizing the pregnant woman with inactivated virus or bacteria vaccines or toxoids. Previously immunized pregnant women who have not received a Td immunization within the last 10 years should receive a booster dose once past the first trimester. Women who are unimmunized or only partially immunized against tetanus should complete as much of the primary series as possible during the last two trimesters of the pregnancy.
Depending on when the woman seeks prenatal care and the required interval between doses, one or two doses of Td can be administered before delivery. Eligible women who do not complete the required three-dose series during pregnancy should be followed up after delivery to assure they receive the doses necessary for protection.

All pregnant women should be evaluated for immunity to rubella. Women susceptible to rubella should be immunized immediately after delivery. In addition, a woman's status as a carrier of hepatitis B should also be assessed during pregnancy. A woman infected with hepatitis B virus should be followed carefully so that her child can receive HBIG and the hepatitis B vaccine series shortly after delivery.

There is no known risk to the fetus from passive immunization of pregnant women with IG. Further information regarding immunization of pregnant women is available in the American College of Obstetricians and Gynecologists Technical Bulletin Number 64, May 1982.

\section{Misconceptions concern- ing contraindications to vaccination}

Some healthcare providers inappropriately consider certain conditions or circumstances contraindications to vaccination. Conditions most often inappropriately regarded as routine contraindications include the following:

- Reaction to a previous dose of DTP vaccine that involved only 
soreness, redness, or swelling in the immediate vicinity of the vaccination site or temperature of $<105^{\circ} \mathrm{F}\left(40.5^{\circ} \mathrm{C}\right)$.

- Mild acute illness with lowgrade fever or mild diarrheal illness in an otherwise well child.

- Current antimicrobial therapy or the convalescent phase of illnesses.

- Prematurity. The appropriate age for initiating immunizations in the prematurely born infant is the usual chronologic age. Vaccine doses should not be reduced for preterm infants.

- Pregnancy of mother or other household contact.

- Recent exposure to an infectious disease.

- Breast-feeding. The only vaccine virus that has been isolated from breast milk is rubella vaccine virus. There is no good evidence that breast milk from women immunized against rubella is harmful to infants.

- A history of nonspecific allergies or relatives with allergies.

- Allergies to penicillin or any other antibiotic, except anaphylactic reactions to neomycin (eg, MMR-containing vaccines) or streptomycin (eg, OPV). None of the vaccines licensed in the United States contain penicillin. - Allergies to duck meat or duck feathers. No vaccine available in the United States is produced in substrates containing duck antigens.

- Family history of convulsions in persons considered for pertussis or measles vaccination..$^{7,8}$

- Family history of sudden infant death syndrome in children considered for DTP vaccination.
- Family history of an adverse event, unrelated to immunosuppression, following vaccination.

\section{Adverse events following vaccination}

Modern vaccines are safe and effective but not completely so. Adverse events have been reported following the administration of all vaccines. These events range from frequent, minor, local reactions to extremely rare, severe, systemic illness, such as paralysis associated with OPV. It is often impossible to establish evidence for cause-and-effect relationships when untoward events occur after vaccination because temporal association alone does not necessarily indicate causation. More complete information on adverse reactions to a specific vaccine may be found in the ACIP recommendations for each vaccine.

The National Vaccine Injury Compensation Program established by the National Childhood Vaccine Injury Act of 1986 requires physicians and other healthcare providers who administer vaccines to maintain permanent immunization records and to report occurrences of certain adverse events to the US Department of Health and Human Services. Recording and reporting requirements took effect on March 21, 1988. Reportable reactions include those listed in the Act for each vaccine $e^{9,10}$ and events specified in the manufacturer's vaccine package insert as contraindications to further doses of that vaccine.
Although there will be one system for reporting adverse events following immunizations in the future, at present there are two separate systems. The appropriate method depends on the source of funding used to purchase the vaccine. Events that occur after receipt of a vaccine purchased with public (federal, state, and/or local government) funds must be reported by the administering health provider to the appropriate local, county, or state health department. The state health department completes and submits the correct forms to CDC. Reportable events that follow administration of vaccines purchased with private money are reported by the healthcare provider directly to the Food and Drug Administration (FDA).

\section{Patient information}

Parents, the responsible caregiver, or adult patients should be informed about the benefits and risks of vaccine in understandable language. Ample opportunity for questions and answers should be provided before each immunization. CDC has developed "Important Information Statements" for use with federally purchased vaccines given in public health clinics, but similar statements have not been universally adopted for the private medicalcare sector.

An Important Information Statement must be developed for each vaccine covered by the $\mathrm{Na}$ tional Childhood Vaccine Injury Act (DTP or component antigens, (continued on page 1215) 



\section{Hay fever sufferers \\ have good reason to be confused}

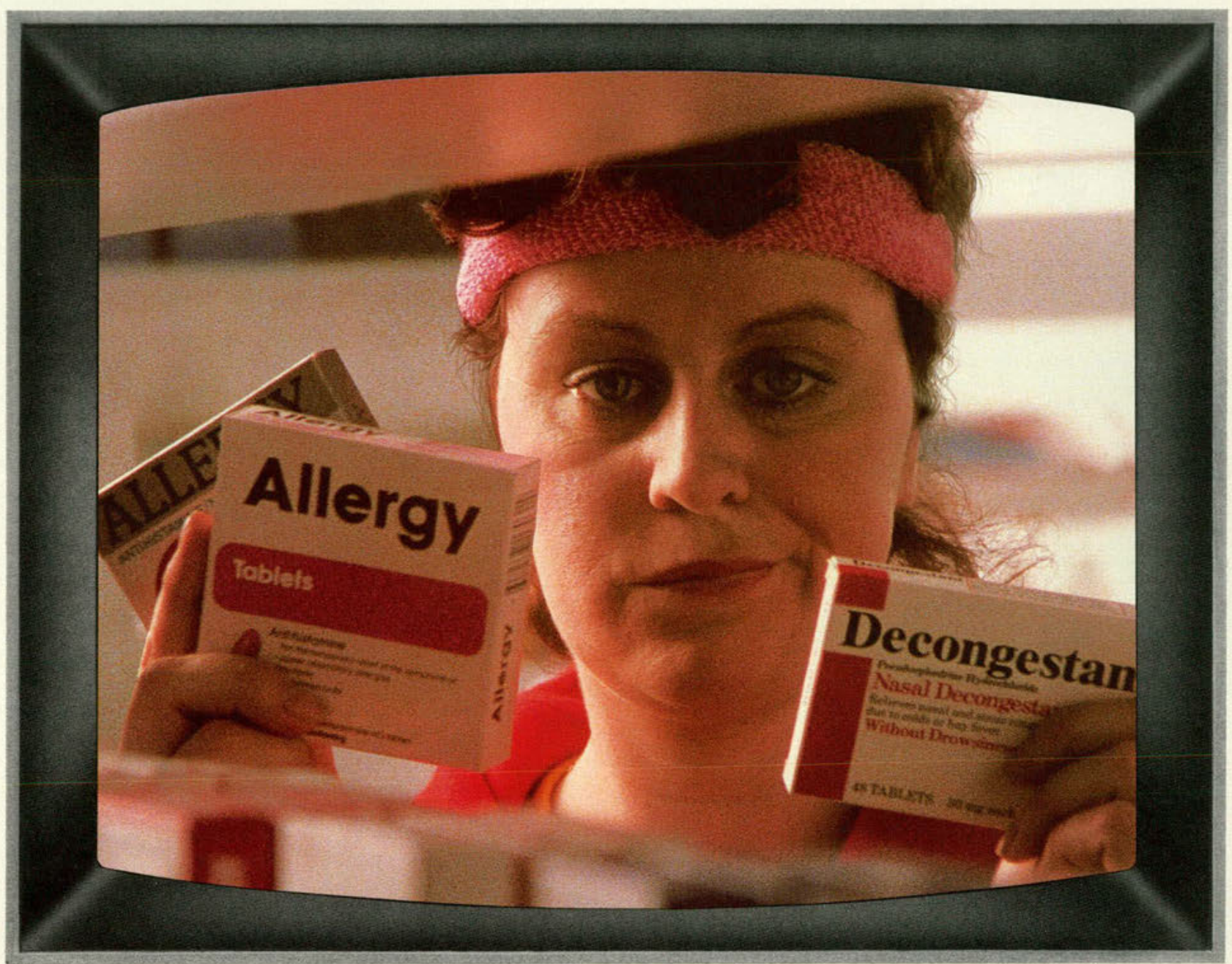

"All those products...all those ingredients...all those claims

-It can be very confusing."

Merrell Dow Pharmaceuticals Inc. nationwide "Allergy Awareness" campaign is advising them to see you.

\section{Only you can choose the best treatment}




\section{Only SELDANE provides fast, unsurpassed relief ${ }^{1.5}$ that lets patients stay alert ${ }^{1,6}$ so they can perform at their best}

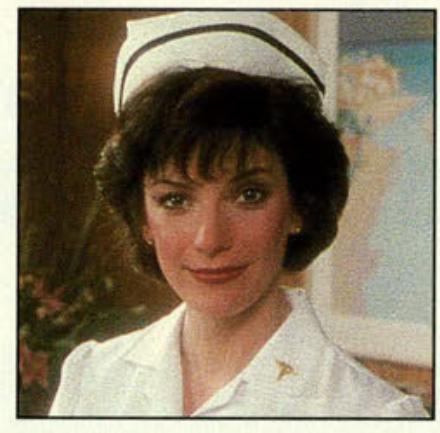

\section{Seldane ${ }^{\circledR}$ (terfenadine) $60 \mathrm{mg}$ Tablets \\ BRIEF SUMMARY}

CAUTION: Federal law prohibits dispensing without prescription

DESCRIPTION

Seldane (terfenadine) is available as tablets for oral administration. Each tablet contains $60 \mathrm{mg}$ terfenadine. Tablets also contain, as inactive ingredients: corn starch, gelatin, lactose, magnesium stearate, and sodium bicarbonate.

\section{INDICATIONS AND USAGE}

Seldane is indicated for the relief of symptoms associated with seasonal allergic rhinitis such as sneezing, rhinorrhea, pruritus, and lacrimation.

CONTRAINDICATIONS

Seldane is contraindicated in patients with a known hypersensitivity to terfenadine or any of its ingreients.

\section{PRECAUTIONS}

Information for patients

Patients taking Seldane should receive the following information and instructions. Antihistamines are prescribed to reduce allergic symptoms. Patients should be questioned about pregnancy or lactation before starting Seldane therapy, since the drug should be used in pregnancy or lactation only if the potential benefit justifies the potential risk to fetus or baby. Patients should be instructed to take Seldane only as needed and not to exceed the prescribed dose. Patients should also be instructed to store this medication in a tightly closed container in a cool, dry place, away from heat or direct sunlight, and away from children.

Carcinogenesis, mutagenesis, impairment of fertility

Oral doses of terfenadine, corresponding to 63 times the recommended human daily dose, in mice for 18 months or in rats for 24 months, revealed no evidence of tumorigenicity. Microbial and micronucleus test assays with terfenadine have revealed no evidence of mutagenesis.

Reproduction and fertility studies in rats showed no effects on male or female fertility at oral doses of up to 21 times the human daily dose. At 63 times the human daily dose there was a small but significant reduction in implants and at 125 times the human daily dose reduced implants and increased post-implantation losses were observed, which were judged to be secondary to maternal toxicity. Pregnancy Category $C$

There was no evidence of animal teratogenicity. Reproduction studies have been performed in rats at doses 63 times and 125 times the human daily dose and have revealed decreased pup weight gain and survival when terfenadine was administered throughout pregnancy and lactation. There are no adequate and well-controlled studies in pregnant women. Seldane should be used during pregnancy only if the potential benefit justifies the potential risk to the fetus.

Nonteratogenic effects

Seldane is not recommended for nursing women. The drug has caused decreased pup weight gain and survival in rats given doses 63 times and 125 times the human daily dose throughout pregnancy and lactation. Effects on pups exposed to Seldane only during lactation are not known, and there are no adequate and well-controlled studies in women during lactation.

Pediatric use

Safety and effectiveness of Seldane in children below the age of 12 years have not been established.

\section{General}

Consideration should be given to potential anticholinergic (drying) effects in patients with lower airway disease, including asthma.

\section{ADVERSE REACTIONS}

Experience from clinical studies, including both controlled and uncontrolled studies involving more than 2,400 patients who received Seldane, provides information on adverse experience incidence for periods of a few days up to six months. The usual dose in these studies was $60 \mathrm{mg}$ twice daily, but in a smal number of patients, the dose was as low as $20 \mathrm{mg}$ twice a day, or as high as $600 \mathrm{mg}$ daily.

In controlled clinical studies using the recommended dose of $60 \mathrm{mg}$ b.i.d., the incidence of reporte adverse effects in patients receiving Seldane was similar to that reported in patients receiving placebo. (See Table below.) ADVERSE EVENTS REPORTED IN CLINICAL TRIALS

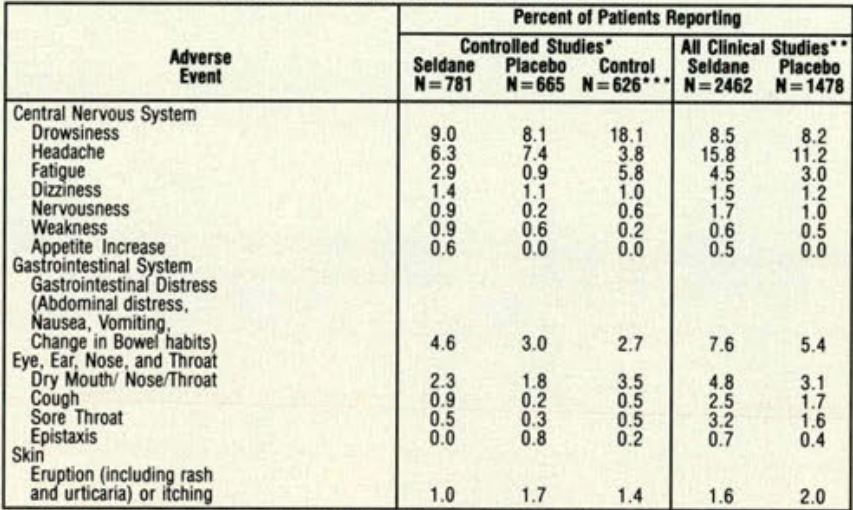

"Duration of treatment in "CONTROLLED STUDIES" was usually 7-14 DAYS.

".Duration of treatment in "ALL CLINICAL STUDIES" was up to 6 months.

"CONTROL DRUGS: Chlorpheniramine (291 patients), d-Chlorpheniramine (189 patients), Clemastine (146

In addition to the more frequent side effects reported in clinical trials (See Table), adverse effects hav been reported at a lower incidence in clinical trials and/or spontaneously during marketing of Seldane that warrant listing as possibly associated with drug administration. These include: alopecia, (hair los or thinning), anaphylaxis, angioedema, arrhythmia (including ventricular tachyarrhythmia), bron chospasm, confusion, depression, galactorrhea, hypotension, insomnia, menstrual disorders (including dysmenorrinea), musculoskeletal symptoms, nightmares, palpitation, paresthesia, photosensitivity, pro longed QT interval, seizures, sweating, syncope, tachycardia, tremor, urinary frequency, and visua disturbances. In clinical trials, several instances of mild, or in one case, moderate transaminase elevations were seen in patients receiving Seldane. Mild elevations were also seen in placebo treated patients. Marketing experiences include isolated reports of jaundice, cholestatic hepatitis, and hepatitis in most cases available information is incomplete. In neither the clinical trials nor marketing experience is a causal relationship of liver abnormalities to Seldane use clear.

OVERDOSAGE

Information concerning possible overdosage and its treatment appears in Full Prescribing Information. DOSAGE AND ADMINISTRATION

The usual dosage for adults and children 12 years and older is $60 \mathrm{mg}$ (1 tablet) twice daily. Product Information as of June, 1988

Division of Merrell Dow Pharmaceuticals inc.

Merrell Dow U.S.A.

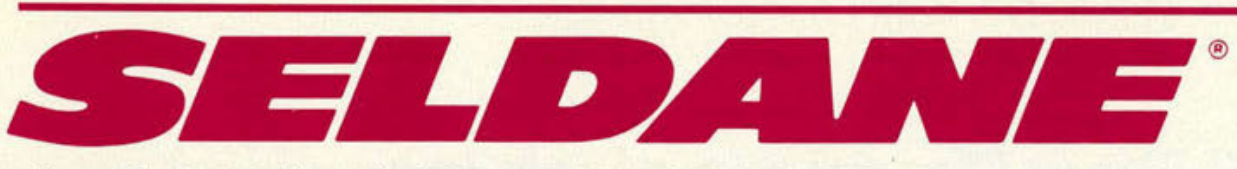

(terfenadine) $60 \mathrm{mg}$ tablets BID for seasonal allergic rhinitis

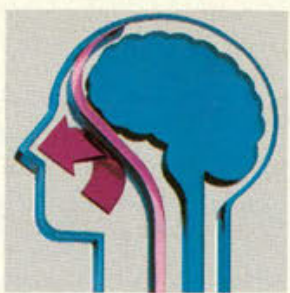

\section{The \# 1 prescribed allergy product in the U.S. and worldwide*}

\footnotetext{
*Based upon worldwide prescription and distribution information (1986-1988) - data on file.

References: 1. Data on file, MERRELL DOW PHARMACEUTICALS INC., Cincinnati, Ohio 45215. 2. Kemp JP, Buckley CE, Gershwin ME, et al: Multicenter, double-blind, placebo-controlled trial of terfenadine in seasonal allergic rhinitis and conjunctivitis. Ann Allergy 1985:54:502-509. 3. Backhouse CI, Brewster BS, Lockhart JDF, et al: Terfenadine

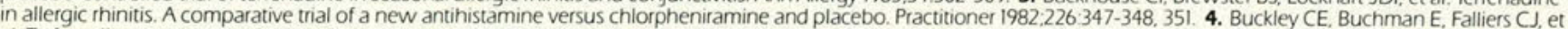
al: Terfenadine treatment of fall hay fever. Ann Allergy 1988,60:123-128. 5. Melillo G. D'Amato G, Zanussi C, et al: A multicentre controlled trial of terfenadine, dexchlorpheniramine, and placebo in allergic rhinitis. Arzneim-Forsch/Drug Res 1982:32:1202-1203. 6. Fink M, Irwin P: CNS effects of the antihistamines diphenhydramine and terfenadine (RMI 9918). Pharmakopsychiat 1979:12:35-44. 7. Roehrs TA Tietz EI, Zorick FJ, et al: Daytime sleepiness and antihistamines. Sleep 1984:7:137-141.
} 

MMR or component antigens, IPV, and OPV). These statements are to be used by all public and private providers of vaccines. Until the Important Information Statements established by the Act become available, the current CDC Important Information Statements should be used in public health clinics and other settings where publicly purchased vaccines are used. The use of similar statements in the private sector is encouraged.

\section{Vaccine programs}

The best way to reduce vaccinepreventable diseases is to have a highly immune population. Universal immunization is an important part of good healthcare and should be accomplished through routine and intensive programs carried out in physicians' offices and in public health clinics. Programs aimed at ensuring that all children are immunized at the recommended ages should be established and maintained in all communities. In addition, appropriate immunizations should be available for all adults.

Every visit to a healthcare provider is an opportunity to update a patient's immunization status with needed vaccines. All adults should complete a primary series of tetanus and diphtheria toxoids, then receive a booster dose every 10 years. Persons $\geqslant 65$ years old and all adults with medical conditions that place them at risk for pneumococcal disease or serious complications of influenza should receive one dose of pneumococcal polysaccharide vac- cine and annual injections of influenza vaccine. In addition, immunization programs for adults should provide MMR vaccine whenever possible to anyone believed susceptible to measles, mumps, or rubella. Use of MMR ensures that the recipient has been immunized against three different diseases and causes no harm if the vaccinee is already immune to one or more of its components.

Official health agencies should take necessary steps, including developing and enforcing school immunization requirements, to assure that students at all grade levels, including college students, and those in childcare centers are protected against vaccine-preventable diseases. Agencies should also encourage institutions such as hospitals and extended-care facilities to adopt policies regarding the appropriate immunization of residents and employees.

Dates of immunization (day, month, and year) should be recorded on institutional immunization records, such as those kept in schools and childcare centers. This will facilitate assessments that a primary vaccine series has been completed according to an appropriate schedule and that needed boosters have been obtained at the correct time.

Tickler or recall systems can identify children who are due for immunizations or are behind schedule so parents can be contacted and reminded to have their children immunized. The ACIP recommends the use of these systems by all healthcare providers. Such systems should also be developed by healthcare providers who treat adults to ensure that at-risk persons receive influenza vaccine annually.

\section{Immunization records}

Documentation of patient immunizations will help ensure that persons in need of vaccine receive it and that adequately vaccinated patients are not overimmunized with increased risk of hypersensitivity (eg, tetanus toxoid hypersensitivity).

\section{Patient's personal records}

Official immunization cards have been adopted by every state and the District of Columbia to encourage uniformity of records and to facilitate the assessment of immunization status by schools and childcare centers. The records are also important tools in immunization education programs aimed at increasing parental and patient awareness of the need for vaccines. A permanent immunization record card should be established for each newborn infant and maintained by the parent. In many states, these cards are distributed to new mothers before discharge from the hospital.

\section{Provider records}

The National Vaccine Injury Compensation Program requires each healthcare provider to record in the vaccine recipient's permanent medical record (or in a permanent office log or file) the provider's name, address, and title (if appropriate), the type of immunobiologic administered, the manufacturer, lot number, and 
date of administration. Healthcare provider is any licensed healthcare professional, organization, or institution, whether private or public (including federal, state, and local departments and agencies), under whose authority a specified vaccine is administered. The vaccines covered under this new law include: DTP and MMR (or any of their components given singly or in combination), OPV, and IPV. A permanent immunization record should also be established and maintained for adults and children who receive vaccines not covered by the National Vaccine Injury
Act. The ACIP recommends use of standard records that note the type, manufacturer, lot number, and date of administration for each immunobiologic administered. Serologic test results for vaccine-preventable diseases, such as those for rubella screening, as well as documented episodes of adverse events, should also be recorded in the vaccine recipient's permanent medical record.

1. ACIP. General recommendations on immunization. $M M W R$ 1983;32:1-17.

2. ACIP. Poliomyelitis prevention: Enhanoed-potency inactivated poliomyelitis vaccine - supplementary statement. MMWR 1987;36:795-798.
3. Kaplan JE, Nelson DB, Schonberger LB, et al: The effect of immune globulin on the response to trivalent oral poliovirus and yellow fever vaccinations. Bull WHO 1984;62:585-590.

4. Herman JJ, Radin R, Schneiderman R: Allergic reactions to measles (rubeola) vaccine in patients hypersensitive to egg protein. $J \mathrm{Pe}$ diatr 1983;102:196-199.

5. Murphy KR, Strunk RC: Safe administration of influenza vaccine in asthmatic children hypersensitive to egg proteins. $J$ Pediatr 1985; 106:931-933.

6. Jacobs RL, Lowe RS, Lanier BQ: Adverse reactions to tetanus toxoid. JAMA 1982;247:40-42. 7. ACIP. Pertussis immunization: Family history of convulsions and use of antipyreticssupplementary ACIP statement. MMWR $1987 ; 36: 281-282$.

8. ACIP. Measles prevention. MMWR 1987:36:409-418, 423-425.

9. CDC. National Childhood Vaccine Injury Act: Requirements for permanent vaccination records and for reporting of selected events after vaccination. MMWR 1988;37-197-200.

10. Food and Drug Administration. New report. ing requirements for vaccine adverse events. FDA Drug Bull 1988; 18(2):16-18.

\section{Sources of vaccine information}

In addition to these general recommendations, elers or persons who provide information to trav-Guide for Adult Immunization (1985) is prothe practitioner can draw on a variety of elers about specific outbreaks of communicable duced by the American College of Physicians sources for specific data and updated informa- diseases abroad. They include health informa- for physicians caring for adults. It emphasizes tion including:

Official vaccine package circulars. Manufacturer-provided product-specific information approved by the FDA with each vaccine. Some of these materials are reproduced in the Physi tion for prevention and specific recommenda- use of vaccines in healthy adults and adults tions for immunization. Memoranda and/or with specific disease problems. It is available placement on mailing list are available from from American College of Physicians, Division Division of Quarantine, Center for Prevention of Scientific Activities, Health and Public PolServices (CPS), CDC, Atlanta, GA 3033 icy, 4200 Pine Street, Philadelphia, PA 19104 cian's Desk Reference (PDR).

Morbidity and Mortality Weekly Report (MMWR). Published weekly by CDC, MMWR contains regular and special ACIP recommendations on vaccine use and statements of vaccine policy as they are developed and reports of specific disease activity. Subscriptions are available through Superintendent of Documents, US Government Printing Office, Washington, DC 2040 Also available through MMS Publications, CSPO Box 9120, Waltham, MA 02254.

Health Information for International Travel. Booklet published annually by CDC as a guide to national requirements and with recommen dations for specific immunizations and health practices for travel to foreign countries. Purchase from the Superintendent of documents (address previously noted).

The Report of the Committee on Infectious Technical bulletins of the American ColDiseases of the American Academy of Pediat- lege of Obstetricians and Gynecologists are rics (Red Book). This report, which contains updated periodically. These bulletins contain recommendations on all licensed vaccines, is up- important information on immunization of pregdated every 2 to 3 years, most recently in 1988. nant women. They are available from AmeriPolicy changes for individual recommendations can College of Obstetricians and Gynecologists, for immunization practices are published as Attention: Resource Center, 40912 th Street needed by the American Academy of Pediat- SW, Washington, DC 20024-2188.

rics in the journal Pediatrics. They are avail-

able from American Academy of Pediatrics, Pub- State and many local health departments lications Division, 141 Northwest Point Blvd, frequently provide technical advice, printed PO Box 927, Elk Grove Village, IL 60009-0927. information on vaccines and immunization

Control of Communicable Diseases in Man is published by the American Public Health Association every 5 years, most recently in 1985 Division of Immunization, CPS, CDC, At(ed 14). The manual contains information about lanta, GA 30333, telephone (404) 639-3311, ofinfectious diseases, their occurrence worldwide, fers technical advice on vaccine recommendadiagnoses and therapy, and up-to-date recom- tions, disease outbreak control, and sources of mendations on isolation and other control meas- immunobiologics. In addition, a course on the ures for each disease presented. It is available epidemiology, prevention, and control of vacAdvisory memoranda are published as from the American Public Health Association, cine-preventable diseases is offered each year needed by CDC to advise international trav- 1015 Fifteenth St NW, Washington, DC 20005 . in Atlanta and, on occasion, in different states. 